\title{
REPORT ON DOE ANALYTICAL
}

\section{LABORATORY CAPACITY}

\section{AVAILABLE TO MEET EM}

\section{ENVIRONMENTAL SAMPLING}

\section{AND ANALYSIS NEEDS \\ FOR FY 93 - 99}

Prepared by:

Analytical Services Division (EM 263 )

Office of Compliance and Program Coordination

Office of Environmental Management

U.S. Department of Energy

November 30,1994 
This report has been reproduced directly from the best available copy.

Available to DOE and DOE Contractors from the Office of Scientific and Technical Information, P.O. Box 62, Oak Ridge, TN 37831; prices available from (615) 576-8401.

Available to the public from the U.S. Department of Commerce, Technology Administration, National Technical Information Service, Springfield, VA 22161, (703) 487-4650. 


\section{REPORT ON DOE ANALYTICAL}

\section{LABORATORY CAPACITY}

\section{AVAILABLE TO MEET EM}

\section{ENVIRONMENTAL SAMPLING}

\section{AND ANALYSIS NEEDS}

\section{FOR FY 93 - 99}

Prepared by:

Analytical Services Division (EN 263 ) Office of Compliance and Program Coordination Office of Environmental Management. U.S. Department of Energy

November 30.1994 


\section{DISCLAIMER}

Portions of this document may be illegible in electronic image products. Images are produced from the best available original document. 


\section{EXECUTIVE SUMMARY}

This report describes and compares the results of two studies conducted by the Office of Environmental Management's (EM's) Analytical Services Division (EM-263): the Department of Energy (DOE) Analytical Laboratory Capacity Study, and the EM Analytical Needs Assessment. These studies were designed to determine the DOE analytical laboratory capacity available to EM, and to provide forecasts of EM sampling and analysis needs. This report matches the data from the two studies, providing a comparison of estimated EM analytical needs to DOE laboratory capacity.

A model was developed in the DOE analytical laboratory capacity study to determine laboratory capacity. The capacity study was based on the time taken to complete a number of laboratory operations making up each major sample analysis. By applying this model at all the major analytical laboratories within the DOE Complex, estimates of the DOE laboratory capacity available to EM were made.

A second model was developed for assessing future EM analytical needs. This model, using EM analytical demand for activities undertaken in fiscal year (FY) 93, was used together with activities planned for FY 94-99 to project future EM analytical needs in this period. Data obtained from this model are considered only tentative in nature, since reports were not received from all the major sites across the DOE Complex, and no account was taken for the fraction of planned activities that will be delayed or will not take place. Data collected from application of these two models across the DOE Complex were entered into databases, and summary data extracted from these databases are presented here.

Based on the data received to this point, Complex-wide EM annual analytical needs are projected to be about one million sample analyses in FY 93, to increase to over three million in FY 94 and $F Y 95$, and to return to around 1.3 million sample analyses in the period FY 96 through FY 99. In comparison, using the same set of standard sample analyses, the currently utilized capacity of laboratories within the DOE Complex is estimated to be about 730,000 sample analyses, of which approximately 390,000 sample analyses are performed for EM. The difference between the EM demand and the DOE capacity is made up by laboratories in the commercial sector.

An estimated theoretical capacity of 1.9 million sample analyses exists within the DOE laboratories. This capacity can potentially be achieved if laboratories receive optimum sample loads. While this number is unlikely to be achieved in practice, improvements in sample management programs could lead to considerably more effective utilization of these laboratories by EM.

Data provided in this report, and available in the EM analytical needs and DOE laboratory capacity databases, will be a critical resource to EM in making key decisions on acquiring the optimum analytical services for EM. 


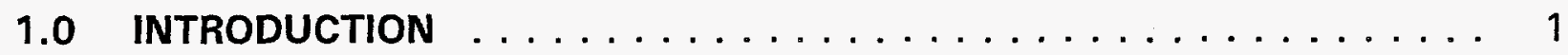

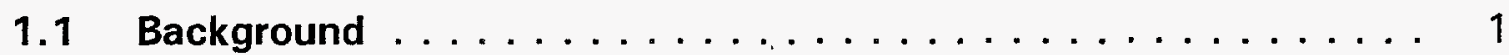

1.2 Scope of Studies $\ldots \ldots \ldots \ldots \ldots \ldots \ldots \ldots \ldots \ldots \ldots \ldots$

2.0 DOE ANALYTICAL LABORATORY CAPACITY STUDY $\ldots \ldots \ldots \ldots 3$

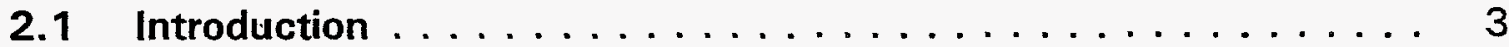

2.2 Model Description . . . . . . . . . . . . . . . . 3

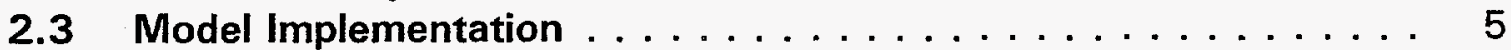

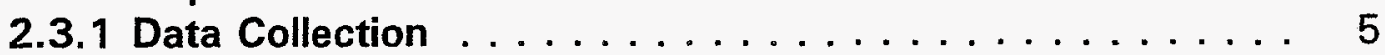

2.3.2 Data Review ................... 9

2.3.3 Data Summarization . . . . . . . . . . . . 11

2.4 Results . . . . . . . . . . . . . . . . . 11

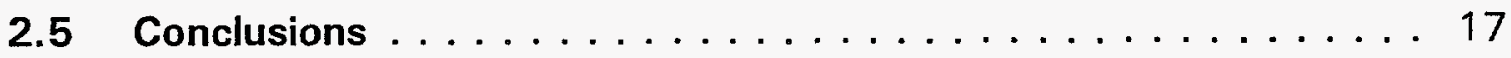

3.0 EM ANALYTICAL NEEDS ASSESSMENT $\ldots \ldots \ldots \ldots \ldots \ldots \ldots \ldots$

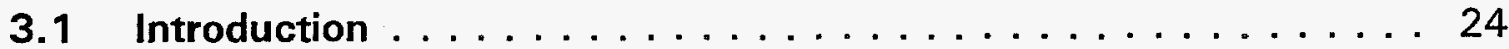

3.2 Model Description . . . . . . . . . . . . . . . . 24

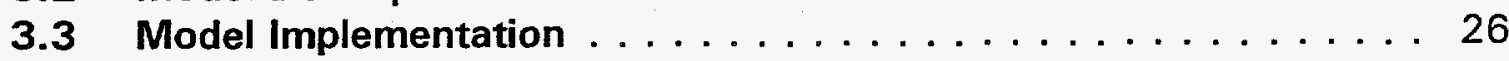

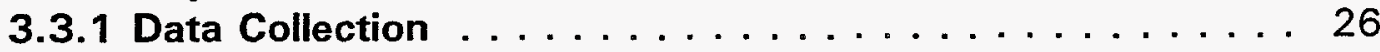

3.3.2 Data Review . . . . . . . . . . . . . . 27

3.3.3 Data Summarization . . . . . . . . . . . . . 27

3.4 Results . . . . . . . . . . . . . . . . . 29

3.5 Conclusions . . . . . . . . . . . . . . . 40

4.0 COMPARISON OF DOE LABORATORY CAPACITY TO EM ANALYTICAL NEEDS PROJECTIONS $\ldots \ldots \ldots \ldots \ldots \ldots \ldots \ldots \ldots \ldots$

4.1 Introduction . . . . . . . . . . . . . . . . 41

4.2 Standardization of Sample Analyses . . . . . . . . . 41

4.3 Comparison of Standardized Sample Analyses . . . . . . . . . 41

4.4 Conclusions . . . . . . . . . . . . . . . . . 47

5.0 OVERALL CONCLUSIONS $\ldots \ldots \ldots \ldots \ldots \ldots \ldots \ldots \ldots \ldots$

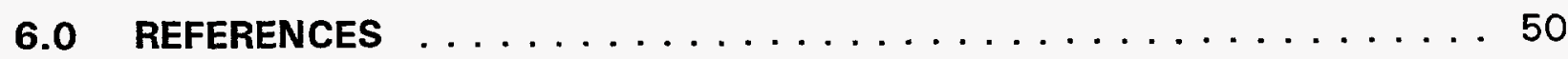




\section{LIST OF APPENDICES}

Appendix A: $\quad$ Estimating DOE Analytical Laboratory Capacity Available to EM Model Description

Appendix B: $\quad$ Estimating DOE Analytical Laboratory Capacity Available to EM Reporting Formats and Instructions

Appendix C: $\quad$ Estimating DOE Analytical Laboratory Capacity Available to EM Operation of Database Spreadsheet System

Appendix D: $\quad$ Estimating DOE Analytical Laboratory Capacity Available to EM Data Tables

Appendix E: Determination of EM Environmental Sampling and Analysis Needs for FY 93-99 - Reporting Formats and Instructions

Appendix F: Determination of EM Environmental Sampling and Analysis Needs for FY 93-99 - Operation of Database Spreadsheet System

Appendix G: Determination of EM Environmental Sampling and Analysis Needs for FY 93-99 - Data Tables 


\section{LIST OF TABLES}

Table I: $\quad$ Participation of DOE Laboratories in the EM-263 Capacity Study . 6

Table II: Total Sample Analyses (in thousands) by Capacity Type and Laboratory . . . . . . . . . . . . . . . . . . . 12

Table III: Distribution of Sample Analyses (in thousands) by RCZ . . . . . 16

Table IV: Distribution of Sample Analyses (in thousands) by Matrix Type . . 16

Table V: Participation of DOE Sites in the EM-263 Needs Assessment . . . 26

Table VI: Total Projected EM Sample Analysis Needs (in thousands) by Fiscal Year and Site . . . . . . . . . . . . . . . . . . . . 29

Table VII: Total Projected EM Sample Needs (in thousands) by Fiscal Year

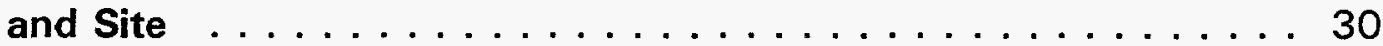

Table VIII: Distribution of EM Sample Analysis Needs (in thousands) by Radiation Level . . . . . . . . . . . . . . . . . . . . . . . . 31

Table IX: Distribution of EM Sample Analysis Needs (in thousands) by Matrix Type . . . . . . . . . . . . . . . . . . . . . 34

Table X: Common Sample Analyses Equivalent to Sample Analyses Used in Estimating DOE Laboratory Capacity . . . . . . . . . . . . 42

Table XI: Common Sample Analyses Equivalent to Sample Analyses Used in Projecting EM Analytical Needs . . . . . . . . . . . . . 43

Table XII: Comparison of DOE Laboratory Capacity and EM Analytical Demand (in Thousands of Sample Analyses) by Sample Analysis Type . . . . . . . . . . . . . . . . . . . . . . . . . 44

Table XIII: Comparison of DOE Laboratory Capacity and EM Analytical Demand (in Thousands of Sample Analyses) by Sample Radiation Level and Matrix Type . . . . . . . . . . . . . . . . . . . . 46

Table XIV: Comparison of DOE Laboratory Capacity and EM Analytical Demand (in Thousands of Sample Analyses) by Regulatory Driver

Table XV: Comparison of DOE Laboratory Capacity and EM Analytical Demand (in Thousands of Sample Analyses) by Laboratory and Site 
Figure 1: Data Input Sheet for Estimating DOE Analytical Capacity . . . . 7

Figure 2: Examples of Output from Summary Data Worksheet for Estimating DOE Analytical Capacity . . . . . . . . . . . . . . 8

Figure 3: Total Annual Capacity in Sample Analyses by Capacity Type . . . 13

Figure 4: Distribution of Sample Analyses by Operations Office . . . . . . . 14

Figure 5: Distribution of Sample Analyses by Laboratory . . . . . . . . . . . 15

Figure 6: Distribution of Sample Analyses by Analytical Protocol . . . . . 18

Figure 7: Distribution of Sample Analyses by Funding Source . . . . . . . 19

Figure 8: Distribution of Utilized Capacity by Sample Analysis Type . . . . 20

Figure 9: Distribution of Existing Capacity by Sample Analysis Type . . . . . 21

Figure 10: Distribution of Upgradable Capacity by Sample Analysis Type . . 22

Figure 11: EM-263 Analytical Needs Model . . . . . . . . . . . . . . . 25

Figure 12: Example of an Activity Sample and Analysis Profile . . . . . . . . 28

Figure 13: Total Projected Annual EM Sample and Sample Analysis Needs for FY 93-99 . . . . . . . . . . . . . . . . . . . 31

Figure 14: Distribution of Sample Analysis Needs by EM Office . . . . . . 32

Figure 15: Distribution of Sample Analysis Needs by Site . . . . . . . . . 33

Figure 16: Distribution of Sample Analysis Needs by Activity . . . . . . . 35

Figure 17: Distribution of Sample Analysis Needs by Regulatory Driver . . . . 36

Figure 18: Distribution of FY 93 Needs by Sample Analysis Type . . . . . . . 37

Figure 19: Distribution of FY 94 Needs by Sample Analysis Type . . . . . . . 38

Figure 20: Distribution of FY 95-99 Needs by Sample Analysis Type . . . . 39 


\section{LIST OF ACRONYMS}

$\begin{array}{ll}\text { AA } & \text { Atomic Absorption } \\ \text { ADS } & \text { Activity Data Sheet } \\ \text { AES } & \text { Atomic Emission Spectroscopy } \\ \text { CERCLA } & \text { Comprehensive Environmental Response, Compensation, and Liability } \\ & \text { Act } \\ \text { CLP } & \text { Contract Laboratory Program } \\ \text { CWA } & \text { Clean Water Act } \\ \text { DOE } & \text { U.S. Department of Energy } \\ \text { EC } & \text { Existing Capacity } \\ \text { EM } & \text { Office of Environmental Management } \\ \text { EM-20 } & \text { Office of Compliance and Program Coordination } \\ \text { EM-263 } & \text { Analytical Services Division } \\ \text { EM-3O } & \text { Office of Waste Management } \\ \text { EM-4O } & \text { Office of Environmental Restoration } \\ \text { EM-5O } & \text { Office of Technology Development } \\ \text { FSMP } & \text { Field Sample Management Program } \\ \text { FY } & \text { Fiscal Year } \\ \text { GC/MS } & \text { Gas Chromatography/Mass Spectrometry } \\ \text { GF } & \text { Graphite Furnace } \\ \text { ICP } & \text { Inductively Coupled Plasma } \\ \text { IPEP } & \text { Integrated Performance Evaluation Program } \\ \text { M\&O } & \text { Management and Operations } \\ \text { MC } & \text { Upgradable Capacity } \\ \text { NSMP } & \text { National Sample Management Program } \\ \text { NSTS } & \text { National Sample Tracking System } \\ \text { OSM } & \text { Office of Sample Management } \\ \text { OU } & \text { Operable Unit } \\ \text { PTS } & \text { Progress Tracking System } \\ \text { RCRA } & \text { Resource Conservation and Recovery Act } \\ \text { RCZ } & \text { Radiation Control Zone } \\ \text { SAIC } & \text { Science Applications International Corporation } \\ \text { SWMU } & \text { Solid Waste Management Unit } \\ \text { TCLP } & \text { Toxicity Characteristics Leaching Procedure } \\ \text { TMOC } & \text { Theoretical Maximum of Operational Cycles } \\ \text { UC } & \text { Utilized Capacity } \\ \text { VOA } & \text { Volatile Organic Analysis } \\ \text { WAG } & \text { Waste Area Group } \\ \text { WPI } & \text { Waste Policy Institute } \\ & \\ & \\ & \\ & \end{array}$




\subsection{INTRODUCTION}

\subsection{Background}

The Department of Energy's (DOE's) Office of Environmental Management (EM) has been tasked with addressing all environmental contamination and waste problems facing the Department. A fundamental element of the EM program is to establish and maintain sufficient analytical capabilities and capacity to effectively support its analytical needs. The Analytical Services Division (EM-263) was therefore established within EM with the primary mission of ensuring that EM operations have the costeffective and high-quality chemical and radiochemical analytical laboratory support needed to produce the required environmental data.

To accomplish its mission, EM-263 set out to collect detailed, current information on both the analytical laboratory capacity available to EM within the DOE Complex, and the planned sampling and analysis needs of EM in the next five years. While attempts had been made in the past to assess overall EM analytical needs, ${ }^{1,2}$ the information collected was either flawed, preliminary in nature, or not current, and no previous studies were conducted to determine DOE analytical laboratory capacity Complexwide. The information collected in the studies described here, when combined with data collected from other programs established within EM-263, such as the National Sample Tracking System (NSTS) ${ }^{3}$ and the Integrated Performance Evaluation Program (IPEP), ${ }^{4}$ will be critical in assisting EM management to:

- Respond to requests from Congress and others for information on EM analytical needs, capacities, and capabilities;

- Provide credible EM sample load forecasts;

- Predict insufficient or surplus analytical laboratory capabilities and capacity;

- Assess laboratory utilization and sample management efficiency;

- Optimize the type and level of commercial laboratory participation in the EM program;

- Evaluate the need for DOE analytical laboratory construction, upgrading, or closure;

- Prioritize method development and training programs; and

- Determine the level and direction of funding necessary to carry out the required sampling and analysis activities.

This report describes the results of two studies conducted by EM-263 in fiscal year 1994 (FY 94): the DOE Analytical Laboratory Capacity Study and the EM Analytical Needs Assessment. These studies were designed to determine the DOE analytical 
laboratory capacity available to EM, and to provide forecasts of EM sampling and analysis needs. This report matches the data from the two studies, providing a comparison of estimated EM analytical needs to DOE laboratory capacity.

\subsection{Scope of Studies}

The studies described in this report were carefully designed to obtain and compare consistent and meaningful data on analytical needs and laboratory capacity. Both studies used a sample analysis as a consistent unit of measurement, and despite the different sources of data in the two studies, a common set of sample analyses was generated for comparing the data. Sample analysis information was collected primarily for chemical analytical operations (i.e. organic, inorganic, and radiochemical operations), though other operations such as the toxicity characteristics leaching procedure (TCLP) and physical measurements are also included.

Requests for participation in the DOE Analytical Laboratory Capacity Study were sent through the Operations Offices to the managers of all the chemical analytical laboratories within the DOE Complex that attend the annual DOE Analytical Managers' meetings. They were asked to complete the study for all analytical operations that are or were potentially available to EM. They were also asked to identify sample analyses that were performed for EM and those that were performed for other organizations within DOE.

Requests for participation in the EM Analytical Needs Assessment were sent via the Operations Offices to representatives of sample management organizations at the ten largest EM sites within the DOE Complex. Information was requested on all sampling and analysis activities funded by the Offices of Waste Management (EM-30), Environmental Restoration (EM-40), and Facility Transition and Management (EM-60) at the ten sites. 


\subsection{DOE ANALYTICAL LABORATORY CAPACITY STUDY}

\subsection{Introduction}

The DOE Analytical Laboratory Capacity Study was conducted to give EM-263 current information about existing and future analytical capacities and capabilities of site laboratories within the DOE Complex. Each DOE site may have one or more analytical laboratories in operation. These facilities were established to support site missions such as production, research and development, and personnel and environmental monitoring. With changing site missions and the DOE directives for environmental monitoring and cleanup, these laboratories are either devoting or planning to devote resources to support EM activities.

The DOE site laboratories represent a considerable amount of capital investment and analytical capability, capacity, and expertise that can be applied to support the EM mission. They not only provide cost-effective high-volume analytical laboratory services, but are also highly recognized analytical research and development centers. Several sites have already transferred their analytical capability from traditional production support to environmental monitoring and waste management support. A model was developed to determine the analytical capacity of all laboratories in the DOE Complex. The model was applied at nearly all the major laboratories and the results collected from these studies are summarized in this report.

\subsection{Model Description}

The DOE laboratory capacity model is a modification of a similar model developed at Hanford for managing analytical capacities at their laboratories. ${ }^{5}$ Its key features are described here, and a full description of the model is given in Appendix A.

The model addresses both analytical capability (the types of analytical measurements a laboratory is able to perform on a given sample type) and analytical capacity (the number of these measurements a laboratory is able to complete in a given time). The results in this report are presented in terms of annual capacities, and three types of annual capacities may be estimated with the model. These are:

- Utilized Capacity (UC) -- the capacity being utilized by the analytical laboratory to support the current work load. This value is used to "calibrate" the model against actual work performed.

- Existing Capacity (EC) -- the analytical capacity that is available with existing personnel levels, existing equipment, and sample loads optimized. This value is of interest to DOE because it represents the total analytical capacity available in the laboratory without expenditures for additional people or equipment. EC capacity estimates include all relevant EM capacity that is now available or might be available when site missions change. 
- Upgradable Capacity (MC) -- the capacity of the analytical laboratory when upgraded to the optimum number of staff for 24 hours/day and seven days/week operation, with the optimum instrumentation added. This value is of interest since it represents the maximum sample analysis capacity of a laboratory without the need for capital building expansion. MC capacity estimates also include all relevant DOE capacity that is now available or might be available when site missions change.

The model estimates the capacity of a laboratory in terms of the numbers of sample analysis cycles completed. A sample analysis cycle is defined as the effort involved in performing a sample analysis on a single customer sample, while a sample analysis is defined as a distinct set of major laboratory operations required to generate specific data for a customer. A given sample analysis can result in the determination of one or more analytes on a customer sample. A list of the major sample analyses typically expected to be performed on EM analytical samples is given in the full description of the model in Appendix A.

A sample analysis usually consists of the following laboratory operations -- sample preparation, instrumental analysis, and data reporting. Therefore, completion of a sample analysis cycle usually requires the completion of three laboratory operational cycles.

The general approach used in estimating DOE Complex analytical laboratory capacity involves first determining a Theoretical Maximum of Operational Cycles (TMOC) for each laboratory operation forming a sample analysis by dividing the time taken to complete one laboratory operational cycle into one year. A series of operational corrections are then applied to the TMOC to account for customer sample analysis time lost or gained due to the following factors:

$\begin{array}{ll}\text { - } & \text { Number of available units } \\ \text { - } & \text { Fquipment availability } \\ \text { - } & \text { Personnel availability } \\ \text { - } & \text { Operation availability } \\ \text { - } & \text { Onstrument calibration } \\ \text { - } & \text { Matrix effects }\end{array}$

Detailed definitions of these correction factors are given in the complete model description in Appendix A. This yields annual capacities for each laboratory operation. The annual capacity of a laboratory for a given sample analysis may then be determined by computing the minimum of the annual capacities for the three laboratory operations making up the sample analysis.

The model also requires that annual capacities for laboratory operations be categorized according to sample handling requirements and matrix type. Five levels of 
requirements for handling samples are defined based on the radiation control zone $(\mathrm{RCZ})$ in which the analytical activity takes place:

$\begin{array}{ll}- & \text { R0 - No radiation containment required } \\ \text { - } & \text { R1 - Bench top or lab hood in RCZ } \\ \text { - } & \text { R2 - Shielded hood in RCZ required } \\ \text { - } & \text { R3 - Glovebox in RCZ required } \\ \text { R4 - Hot cell in RCZ required }\end{array}$

The model defines three levels of sample matrix difficulty:

- M1 - Customer sample matrix typically requiring only one laboratory operational cycle per customer sample processed, e.g., water.

- M2 - Customer sample matrix requiring on average two or less laboratory operational cycles per customer sample processed, e.g., soil, sediment, rock, air, biota.

- M3 - Customer sample matrix requiring on average more than two laboratory operational cycles per customer sample processed, e.g., sludge, waste, pure product, debris.

Such classification of the annual capacities is required in the model, since working with samples in radiation control zones will adversely affect the number of analytical operations that personnel can do in a given time, and the number of times that a major analytical operation may be repeated is a function of the difficulty that the sample matrix presents. Some laboratories did not distinguish between RO and R1 radiation control zones and hence an additional category, RO/R1, was used for presenting such capacity data.

\subsection{Model Implementation}

The model was implemented in the following three stages:

- Capacity data collection from DOE laboratories;

- Data review and refinement; and

- Complex-wide summarization of the data.

\subsubsection{Data Collection}

The capacity model, together with instructions for completing the reporting forms, was distributed to the DOE laboratories listed in Table I. Laboratories were requested to report their capacity data electronically on Lotus 1-2-3 ${ }^{{ }^{*}}$ spreadsheets. Input of all the critical data for computing the requested sample analysis capacities was performed using the spreadsheet shown in Figure 1. Summary of this data and calculations of correction factors and annual capacities based on the model were

* Lotus $1-2-3^{\circledast}$ is a registered trademark of the Lotus Development Corporation. 
Table 1: $\quad$ Participation of DOE Laboratories in the EM-263 Capacity Study

\begin{tabular}{|c|c|c|c|c|c|}
\hline Ops Office & Laboratory & $\begin{array}{c}\text { Data } \\
\text { Received }\end{array}$ & $\begin{array}{c}\text { Data } \\
\text { Reviewed }\end{array}$ & $\begin{array}{l}\text { Response } \\
\text { Received }\end{array}$ & Comments \\
\hline ALBUQUERQUE & Grand Junction & $\overline{\mathrm{Y}}$ & $\overline{\mathrm{Y}}$ & $\mathrm{N}$ & \\
\hline ALBUQUERQUE & Los Alamos Nat'I Lab & $\bar{Y}$ & $\overline{\mathrm{Y}}$ & $\mathrm{N}$ & \\
\hline CHICAGO & Argonne East & $\bar{Y}$ & $\overline{\mathrm{Y}}$ & $\mathbf{N}$ & \\
\hline CHICAGO & Argonne West & $\bar{Y}$ & $\mathbf{N}$ & $\mathbf{N}$ & Only UC data received \\
\hline CHICAGO & Brookhaven Nat'I Lab & $\bar{Y}$ & $\overline{\mathrm{Y}}$ & $\mathrm{N}$ & \\
\hline FERNALD & Fernald & $\bar{Y}$ & $\mathbf{N}$ & $\mathrm{N}$ & \\
\hline IDAHO & Idaho Nat'I Eng Lab - ACU & $\bar{Y}$ & $\bar{Y}$ & $\mathrm{~N}$ & \\
\hline IDAHO & Idaho Nat'I Eng Lab - B\&W & $\bar{Y}$ & $\overline{\mathrm{Y}}$ & $\mathbf{N}$ & \\
\hline IDAHO & Idaho Nat'I Eng Lab - WINCO & $\bar{Y}$ & $\mathrm{~N}$ & $N$ & No UC data received \\
\hline IDAHO & West Valley Nuclear Services & $\bar{Y}$ & $\bar{Y}$ & $\mathbf{N}$ & \\
\hline NEVADA & Nevada Test Site & $\bar{Y}$ & $\bar{Y}$ & $\mathrm{Y}$ & \\
\hline OAK RIDGE & Oak Ridge - K25 & $\bar{Y}$ & $\bar{Y}$ & $\mathbf{N}$ & \\
\hline OAK RIDGE & Oak Ridge Nat'/ Lab & $\bar{Y}$ & $\mathrm{Y}$ & $\mathbf{N}$ & \\
\hline OAK RIDGE & Oak Ridge - Y12 & $\bar{Y}$ & $\bar{Y}$ & $\mathrm{Y}$ & \\
\hline OAK RIDGE & Paducah & $\bar{Y}$ & $\bar{Y}$ & $\mathbf{N}$ & \\
\hline OAK RIDGE & Portsmouth & $\bar{Y}$ & $\overline{\mathrm{Y}}$ & $\mathbf{N}$ & \\
\hline RICHLAND & Hanford - WHC $222 S$ & $\bar{Y}$ & $\bar{Y}$ & $\mathrm{Y}$ & \\
\hline RICHLAND & Hanford - PNL 325 & $\bar{Y}$ & $\bar{Y}$ & $\mathbf{N}$ & \\
\hline ROCKY FLATS & Rocky Flats - $123 \mathrm{Lab}$ & $\bar{Y}$ & $\bar{Y}$ & $\mathbf{N}$ & \\
\hline ROCKY FLATS & Rocky Flats - GL881 & $\bar{Y}$ & $\overline{\mathrm{Y}}$ & $\mathrm{N}$ & \\
\hline ROCKY FLATS & Rocky Flats - RF PA Lab & $\bar{Y}$ & $\bar{Y}$ & $\mathbf{N}$ & \\
\hline SAVANNAH RIVER & WSRC-ALD-CLAB & $\bar{Y}$ & $\bar{N}$ & $\mathbf{N}$ & \\
\hline SAVANNAH RIVER & WSRC-ALD-320M & $\mathbf{Y}$ & $\mathbf{N}$ & $\mathbf{N}$ & \\
\hline SAVANNAH RIVER & WSRC-ALD Water & $\bar{Y}$ & $\bar{N}$ & $\mathbf{N}$ & \\
\hline SAVANNAH RIVER & WSRC-ALD-ETF & $\mathbf{Y}$ & $\mathbf{N}$ & $\bar{N}$ & \\
\hline SAVANNAH RIVER & WSRC-ALD-Tritium & $\bar{Y}$ & $\mathrm{~N}$ & $\mathrm{~N}$ & \\
\hline SAVANNAH RIVER & WSRC-ALD-ITP & $\bar{Y}$ & $\mathbf{N}$ & $\mathbf{N}$ & \\
\hline SAVANNAH RIVER & WSRC-SRTC-ADS & $\bar{Y}$ & $\mathrm{~N}$ & $\mathrm{~N}$ & \\
\hline SAVANNAH RIVER & WSRC-SRTC-DWPT & $\overline{\mathrm{Y}}$ & $\mathbf{N}$ & $\mathbf{N}$ & \\
\hline OAKLAND & Lawrence Livermore Nat'' Lab & $\mathrm{N}$ & $\mathrm{N}$ & $\mathbf{N}$ & No data received \\
\hline ALBUQUERQUE & \begin{tabular}{|l|} 
Kansas City Plant \\
\end{tabular} & $\bar{N}$ & $\bar{N}$ & $\mathbf{N}$ & Model completed but no data received \\
\hline ALBUQUERQUE & Mound & $\bar{N}$ & $\bar{N}$ & $\mathbf{N}$ & No longer in DOE Complex \\
\hline ALBUQUERQUE & Waste Isolation Pilot Plant & $\bar{N}$ & $\mathrm{~N}$ & $\mathrm{~N}$ & No data received \\
\hline
\end{tabular}

automatically performed using macros in a summary spreadsheet. Examples of output from this summary datasheet are presented in Figure 2. A copy of the reporting formats and instructions package sent to the participants is given in Appendix B.

Capacity data were requested for each of the three types of capacity defined in the model and for each radiation control zone in the laboratory. Users were asked to enter the radiation levels of samples permitted in the five radiation control zones defined in the model. Estimated percentages of each sample matrix type analyzed within each sample analysis were also requested in order for the model to calculate a "combined" capacity. This combined capacity represents the capacity for the laboratory based on the operational parameters input for the assumed mix of sample types. A distribution of the protocols used for each sample analysis was also collected. 


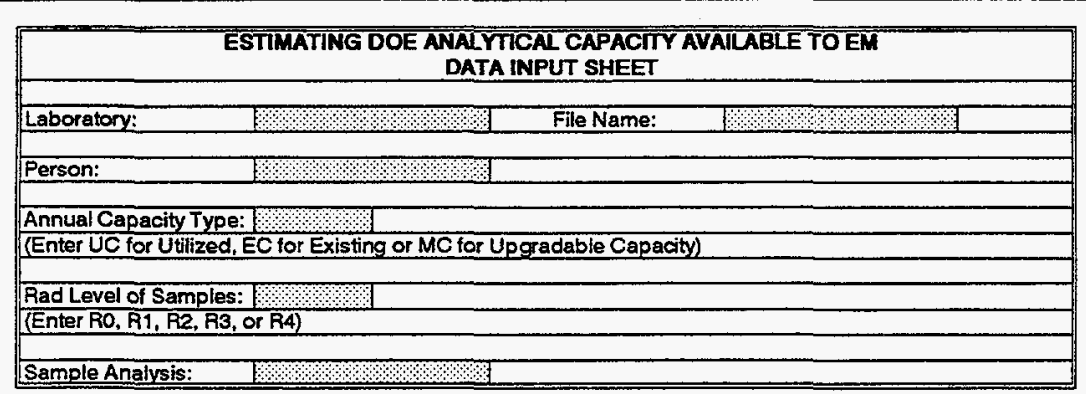

\begin{tabular}{|c|c|c|c|c|c|c|c|c|}
\hline \multicolumn{9}{|c|}{ DISTRIBUTION OF CUSTOMEA SAMPLES ( $(x)$} \\
\hline & \multicolumn{5}{|c|}{ Regulatory-Driven } & \multicolumn{3}{|c|}{ Non-Regulatory-Driven } \\
\hline $\begin{array}{l}\text { Protocol/ } \\
\text { Matrix }\end{array}$ & CWA & CLP & SW846 & NIOSH & Other Reg & \begin{tabular}{c|} 
Waste \\
Treatment
\end{tabular} & $\begin{array}{l}\text { Prod'n } \\
\text { Support }\end{array}$ & $\begin{array}{c}\text { Other } \\
\text { Non-Reg }\end{array}$ \\
\hline M1 & & & & & & & & \\
\hline$M 2$ & & & & & & ৮৮ & & \\
\hline$\overline{M 3}$ & 10 & & & 12 & & ? & & \\
\hline$F M$ & & & & & . & & & \\
\hline
\end{tabular}

\begin{tabular}{|c|c|c|c|}
\hline INPUT DATA FOR AEGULATORY-DRIVE & ALYSES & & \\
\hline \# Customer Sample Analyses in past year: & & & \\
\hline Capacity Parameters/Lab Operation & Prep'n & Ins Anal & Reporting \\
\hline Avg \# Customer Sample Cycles per Week: & & & \\
\hline Avg \# CC Sample Cycles per Week: & & & \\
\hline Avg \# Callibration Sample Cycles per Week: & & & \\
\hline Operation Cycle Time (mins): & & & \\
\hline Calibration Cycle Time (mins): & & & \\
\hline Avg Operating Time (hrs/wlk): & & & \\
\hline Facility Down Time (days/yr): & & & \\
\hline Equipment Down Time (days/yr): & & & \\
\hline Time Personnel Scheduled (hrs/wk): & & & \\
\hline Time Personnel Inetficient (hrs/wh): & & & \\
\hline Time Personnel Unavailable (hrsiwh): & & & \\
\hline Batch Factor: & ২০ & & \\
\hline \# of Instruments or Batch Units: & & & \\
\hline Avg \# of Op'n Cycles per M1 Sample: & & & \\
\hline Avg \# of Op'n Cycles per M2 Sample: & & & \\
\hline Avg \# of Op'n Cycles per M3 Sample: & & & \\
\hline
\end{tabular}

\begin{tabular}{|c|c|c|c|}
\hline \multicolumn{4}{|c|}{ INPUT OATA FOA NON-REGULATORY-DFIVEN SAMPLE ANAIYSES } \\
\hline \multicolumn{4}{|c|}{ \# Customer Sample Analyses in past year: $\quad \checkmark /$ } \\
\hline Capacity Parameters|Lab Operation & Prep'n & Ins Anal & Reporting \\
\hline \\
\hline \multirow{2}{*}{\multicolumn{4}{|c|}{$\begin{array}{l}\text { Avg \# CC Sample Cycles per Woek: } \\
\text { Avg \# Calibration Sample Cycles per Week: }\end{array}$}} \\
\hline & & & \\
\hline \multirow{2}{*}{\multicolumn{4}{|c|}{$\begin{array}{l}\text { Operation Cycle Time (mins): } \\
\text { Calibration Cycle Time (mins): }\end{array}$}} \\
\hline & & & \\
\hline \multicolumn{4}{|l|}{ Avg Operating Time (hrs/wh): } \\
\hline \multicolumn{4}{|l|}{ Facility Down Time (days/yr): } \\
\hline \multicolumn{4}{|l|}{ Equipment Down Time (days/yr): } \\
\hline \multicolumn{4}{|l|}{ Time Personnel Scheduled (hrs/wk): } \\
\hline \multirow{2}{*}{\multicolumn{4}{|c|}{$\begin{array}{l}\text { Time Personnel Inefficient (hrshwh): } \\
\text { Time Personnel Unavailible (hrs/wk): }\end{array}$}} \\
\hline & & & \\
\hline \multicolumn{4}{|l|}{ Batch Factor: } \\
\hline \multicolumn{4}{|l|}{ \# of Instruments or Batch Units: } \\
\hline \multicolumn{4}{|l|}{ Avg \# of Op'n Cycies per M1 Sample: } \\
\hline Avg \# of Op'n Cycles per M2 Sample: & & & \\
\hline Avg \# of Op'n Cycles per M3 Sample: & & & \\
\hline
\end{tabular}

Figure 1: Data Input Sheet for Estimating DOE Analytical Capacity 

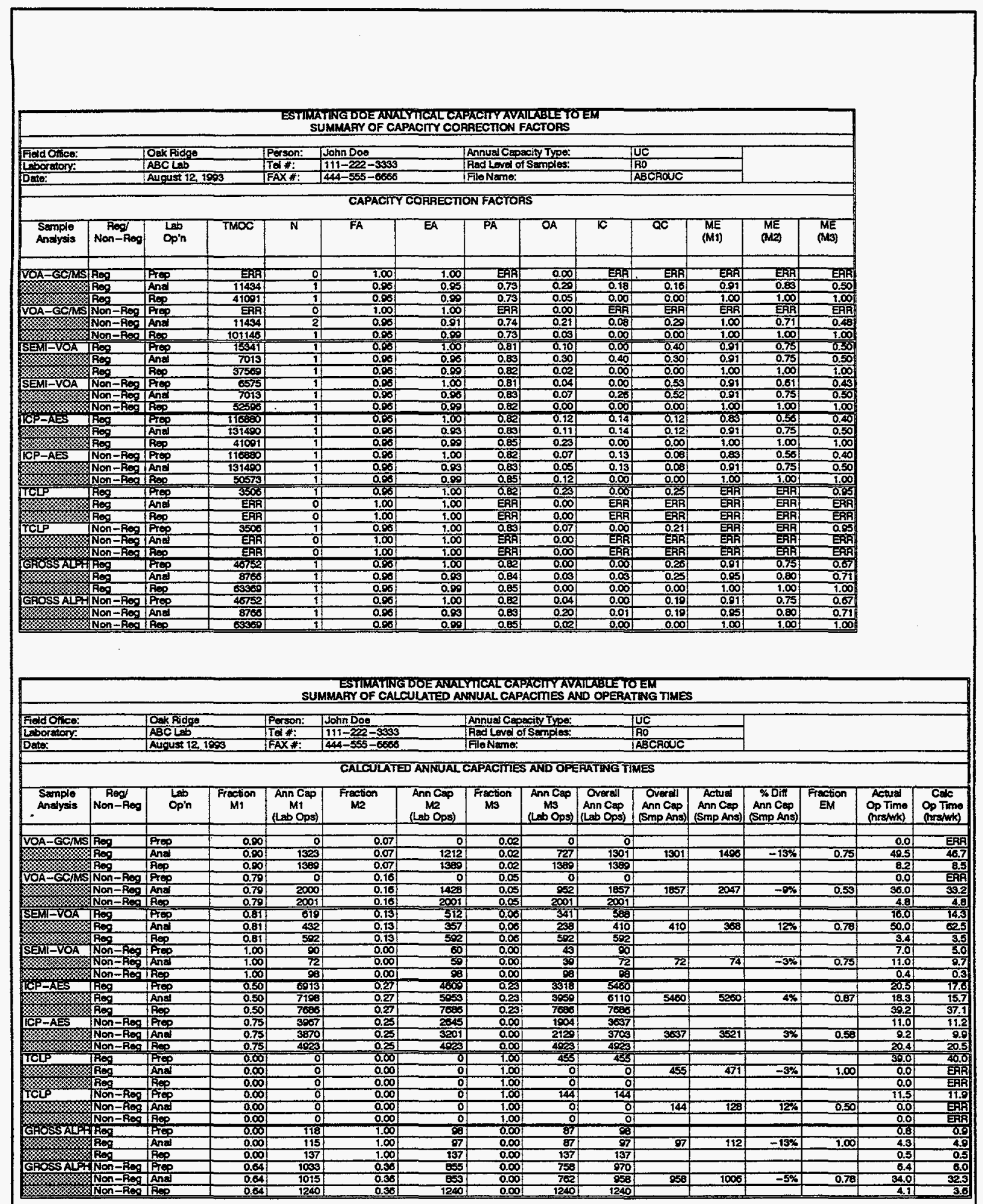

Figure 2: Examples of Outputs from Summary Data Worksheet for Estimating DOE Analytical Capacity 


\subsubsection{Data Review}

With the exception of data received very late in the data collection process, all data received from the laboratories were reviewed for consistency and completeness. The following steps were used in verifying the laboratory capacity data:

1. A minimum of $25 \%$ of the UC data input worksheets were examined from each submission to ensure that the following criteria were met:

A. The table showing the distribution of customer samples is complete in that the sum of percentages adds up to $100 \%$, and the fraction of EM samples is less than or equal to the fraction of total samples for each protocol;

B. The preparation, instrument analysis, and reporting columns of the tables of input data for both regulatory-driven and non-regulatory-driven sample analyses are complete when applicable;

C. The average number of customer sample cycles per week reported is equal to or greater than the number of customer sample analyses reported in the past year divided by 52 ;

D. The cycle times entered are within $\pm 50 \%$ of values established from pilot and standard methods;

E. The average operating times entered are within $\pm 30 \%$ of the products of the numbers of sample cycles and the cycle times; and

F. The average number of operation cycles reported per M3 sample is greater than or equal to the number per $\mathrm{M} 2$ cycle which in turn is greater than or equal to the number per M1 cycle.

2. A minimum of $20 \%$ of the EC data input worksheets were examined from each submission to ensure that all the criteria for the UC data input sheets were met and that the batch factors entered were within $\pm 50 \%$ of those expected from standard laboratory practice.

3. A minimum of $10 \%$ of the MC data input worksheets were examined from each submission to ensure that all the criteria for the EC data input sheets were met and that the number of instruments or batch units entered was no more than a factor of two greater than the number entered in the corresponding UC data input worksheet.

4. A minimum of $25 \%$ of the UC summary data worksheets were examined from each submission to ensure that the following criteria were met: 
A. The calculated overall utilized annual capacities agree with the actual annual capacities to within $\pm 30 \%$ for each sample analysis.

B. The calculated operating times agree with the actual operating times to within a factor of three for each sample analysis.

C. Sample preparation is not the limiting laboratory operation of a sample analysis when estimating the laboratory capacity, except for the following sample analyses:

- $\quad$ organic semi-voa, pcb, herbicide, and pesticide sample analyses;

- $\quad$ radiochemistry alpha spectroscopy; and

- $\quad$ other special sample analyses (e.g. those performed in R3 and R4 RCZs).

D. Data reporting is not the limiting laboratory operation of a sample analysis when estimating the laboratory capacity, except when the sample analysis data reported form part of a full Contract Laboratory Program (CLP) and SW-846 data package.

5. A minimum of $20 \%$ of the EC summary data worksheets were examined from each submission to ensure that criteria B-D for the UC summary data sheets were met and that the values for EC were not greater than five times the values for UC.

6. A minimum of $10 \%$ of the $M C$ summary data worksheets were examined from each submission to ensure that criteria B-D for the UC summary data sheets were met and that the values for MC were not greater than 15 times the values for UC.

All items of concern for which the above criteria were not met were documented, discussed with the appropriate laboratory personnel, and sent to them with a request to review the points of concern raised and either explain the apparent discrepancy or provide more consistent or complete data. Any changes made to the capacity data by the laboratories as a result of the reviewer's comments were resubmitted to EM-263.

Table I shows the laboratories that submitted data early enough for review and those that provided a response to the reviewer's comments. The capacity data used for compiling this report are the best available data obtained from the laboratories. Note: because revised capacity data were received for only three laboratories, not all data used to prepare this report met the criteria given above. The results should be used accordingly. 


\subsubsection{Data Summarization}

The best available capacity data received from the laboratories were summarized using a set of Lotus 1-2-3 ${ }^{\circledR}$ spreadsheets. Macros in the spreadsheets were used to generate a series of database files, convert all sample analysis names used by the laboratories into a consistent set of sample analysis names, and prepare a set of tables of summarized capacity data for the DOE Complex. A detailed description of the data summarization spreadsheet system is given in Appendix $C$ and the summarized tables of capacity data are given in Appendix D. All results presented in the following section of the report were abstracted from data collected in Appendix D.

\subsection{Results}

An overall summary of the analytical capacity available to EM within DOE laboratories is given in Table II. It presents actual, utilized, existing, and upgradable annual capacities for each laboratory in units of sample analyses. The percentage differences between the actual capacities of the laboratories during the past year and their utilized capacities computed using the model are also given. These differences should ideally be equal to zero if the model is correctly calibrated. In most cases, the difference lies below $15 \%$, except for several laboratories under the Chicago and Savannah River Operations Offices. The laboratories were asked to ensure that these differences were less than $25 \%$ before proceeding to estimate $E C$ and $M C$ values. On average over the whole DOE Complex, the computed utilized capacity is $18 \%$ lower than the actual capacity; this provides an overall confidence level in the numbers given in this report. It may also be noted from the data that there is a systematic bias for UC values being lower than corresponding $A C$ values for most of the participating laboratories. This is probably due to a minor flaw in the model, and a detailed analysis of the parameters and equations that make up the model is necessary before developing the next revision.

Overall computed annual capacities for the DOE Complex are shown in Figure 3. The Complex-wide utilized capacity is about 700,000 sample analyses and this value increases by a factor of 2.6 to nearly 1.9 million for the existing capacity. This increase of nearly 1.2 million sample analyses represents the additional capacity potentially available if sample loads sent to the laboratories are optimized. While this can only be obtained in the ideal case, it is clear that fine tuning of the numbers and types of samples sent to laboratories by sample management programs in the Complex could lead to dramatic increases in laboratory capacity without any additional investments in personnel, equipment or facilities.

Complex-wide upgradable capacity equals approximately 5.3 million sample analyses. This represents a factor of 3.2 increase over the EC value and a factor of 8.4 increase over the UC value. While MC values are considerably less reliable than the other capacity values provided here, they do yield an order of magnitude of the potential analytical capacity that could be made available to EM from the DOE laboratories if they are upgraded with optimum instrumentation and continuous staffing. 
Table II: Total Sample Analyses (in thousands) by Capacity Type and Laboratory

\begin{tabular}{|c|c|c|c|c|c|c|c|c|}
\hline Ops Office - Lab/Capacity & $\overline{U C}$ & EC & $\overline{M C}$ & $E C-U C$ & MC-UC & $M C-E C$ & $\overline{A C}$ & $\begin{array}{l}U C-A C \\
\text { pet diff }\end{array}$ \\
\hline \multicolumn{9}{|l|}{ ALBUQUERQUE } \\
\hline Grand Junction & 101 & 54 & 109 & 44 & 99 & 55 & 10 & $-2 \%$ \\
\hline Los Alamos Nat'I Lab & 37 & 34 & 277 & -3 & 241 & 243 & 36 & $1 \%$ \\
\hline \multirow[t]{3}{*}{ TOTALAL } & 47| & 88 & 386 & 41 & 339 & 298 & 47 & $0 \%$ \\
\hline & & & & & & & & \\
\hline & \multicolumn{8}{|c|}{ CHICAGO } \\
\hline Argonne East & 5 & 13 & 70 & 8 & 65 & 57 & 5| & $-7 \%$ \\
\hline Argonne West & 2 & 0 & 0 & -2 & -2 & 0 & 3 & $-42 \%$ \\
\hline Brookhaven Nat'I Lab & 12 & a & 0 & -12 & -12 & 0 & 20 & $-41 \%$ \\
\hline \multirow[t]{2}{*}{ TOTALCH } & $18 !$ & 13 & 70 & 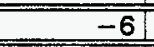 & $52 !$ & 57 & 28 & $-36 \%$ \\
\hline & & & & & & & & \\
\hline \multicolumn{9}{|l|}{ FERNALD } \\
\hline Fernald & 39 & 85 & 254 & 46 & 215 & 169 & 45 & $-14 \%$ \\
\hline \multirow{2}{*}{ TOTALFS } & 39 & 85 & 254 & 46 & $215 \mid$ & 169 & 45 & $-14 \%$ \\
\hline & & & & & & & $=$ & \\
\hline \multicolumn{9}{|l|}{ IDAHO } \\
\hline Idaho Nat'I Eng Lab - ACU & 01 & 1 & 5 & 1 & 5 & 4 & 0 & $-2 \%$ \\
\hline Idaho Nat'I Eng Lab - B\&W & 1 & 10 & 0 & 9 & -1 & -10 & 2 & $-10 \%$ \\
\hline Idaho Nat'I Eng Lab - WINCO & 0 & 25 & 58 & 25 & 58 & 33 & 0 & ERR \\
\hline West Valley Nuclear Services & 4 & 4 & 4 & 0 & -0 & -0 & 4 & $-0 \%$ \\
\hline \multirow{2}{*}{ TOTAL ID } & 61 & 41 & 67 & 34 & 61 & 26 & 71 & $-3 \%$ \\
\hline & & & & & & & 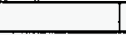 & \\
\hline \multicolumn{9}{|l|}{ NEVADA } \\
\hline Nevada Test Site & 291 & 39 & 71 & 101 & 421 & 32 & 38 & $-23 \%$ \\
\hline \multirow[t]{2}{*}{ TOTALNV } & 29 & 39| & $71]$ & 10 & $42 !$ & 32 & 38 & $-23 \%$ \\
\hline & & & & & & & & \\
\hline \multicolumn{9}{|l|}{ OAK RIDGE } \\
\hline Oak Ridge - K25 & 41 & 80 & 325 & 39 & 284 & 245 & 46 & $-10 \%$ \\
\hline Oak Ridge Nat'l Lab & 23 & 28 & 45 & 5 & 22 & 17 & 24 & $-4 \%$ \\
\hline Oak Ridge - Y12 & 221 & 312 & 1031 & 91 & 811 & 719 & 227 & $-3 \%$ \\
\hline Paducah & 60 & 60 & 152 & -0 & 92 & 92 & 63 & $-5 \%$ \\
\hline Portsmouth & 80 & 86 & 203 & 6 & 124 & 117 & 94 & $-15 \%$ \\
\hline \multirow[t]{2}{*}{ TOTALOR } & 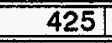 & 5661 & 1757 & 142| & 1332 & 1191 & 453 & $-6 \%$ \\
\hline & & & & & & & & \\
\hline \multicolumn{9}{|l|}{ RICHLAND } \\
\hline Hanford - WHC 222S & 35 & 632 & 1452 & 597 & 1417 & 820 & 36 & $-2 \%$ \\
\hline Hanford - PNL 325 & 8 & 63 & 273 & 56 & 266 & 210 & 8 & $-5 \%$ \\
\hline \multirow[t]{2}{*}{ TOTAL RL } & 43 & 695| & 1725 & 6521 & \begin{tabular}{|c|}
1683 \\
\end{tabular} & 1030 & $44 !$ & $-3 \%$ \\
\hline & & & & & & & & \\
\hline \multicolumn{9}{|l|}{ ROCKY FLATS } \\
\hline \multirow{4}{*}{$\begin{array}{l}\text { Rocky Flats - } 123 \text { Lab } \\
\text { Rocky Flats - GL881 } \\
\text { Rocky Flats - RF PA Lab } \\
\text { TOTAL RF }\end{array}$} & 39 & 226 & 591 & 187 & 552 & 365 & 39 & $-0 \%$ \\
\hline & 13 & 14 & 80 & 2 & 68 & 66 & 12 & $2 \%$ \\
\hline & 6 & 17 & 95 & 11 & 89 & 79 & 6 & $-6 \%$ \\
\hline & 581 & 257 & 767 & 199 & 709 & 510 & 58 & $-0 \%$ \\
\hline & & & & & & & 1 & \\
\hline SAVANNAH RIVER & & & & & & & & \\
\hline WSRC-ALD-CLAB & 6 & 21 & 729 & 14 & 723 & 708 & 12 & $-46 \%$ \\
\hline WSRC-ALD-320M & 0 & 1 & 0 & 1 & 0 & -1 & 0 & $-100 \%$ \\
\hline WSRC-ALD Water & 6 & 9 & 23 & 3 & 16 & 14 & 30 & $-79 \%$ \\
\hline WSRC-ALD-ETF & 1 & 0 & 0 & -1 & -1 & 0 & 12 & $-91 \%$ \\
\hline WSRC-ALD - Tritium & 1 & 3 & 3 & 1 & 1 & 0 & 39 & $-97 \%$ \\
\hline WSRC-ALD -ITP & 1 & 0 & 0 & -1 & -1 & 0 & 1 & $-1 \%$ \\
\hline WSRC-SRTC-ADS & 23 & 41 & 112 & 18 & 90 & 71 & 40 & $-43 \%$ \\
\hline WSRC-SRTC-DWPT & 5 & 8 & 8 & 3 & 3 & 0 & 12 & $-59 \%$ \\
\hline TOTAL SA & 44 & 83 & 875 & 39 & 831 & 792 & 146 & $-70 \%$ \\
\hline & & & & & & & & \\
\hline TOTAL COMPLEX & 708 & 1866 & 5973 & 1158 & 5265 & 4107 & 865 & $-18 \%$ \\
\hline
\end{tabular}




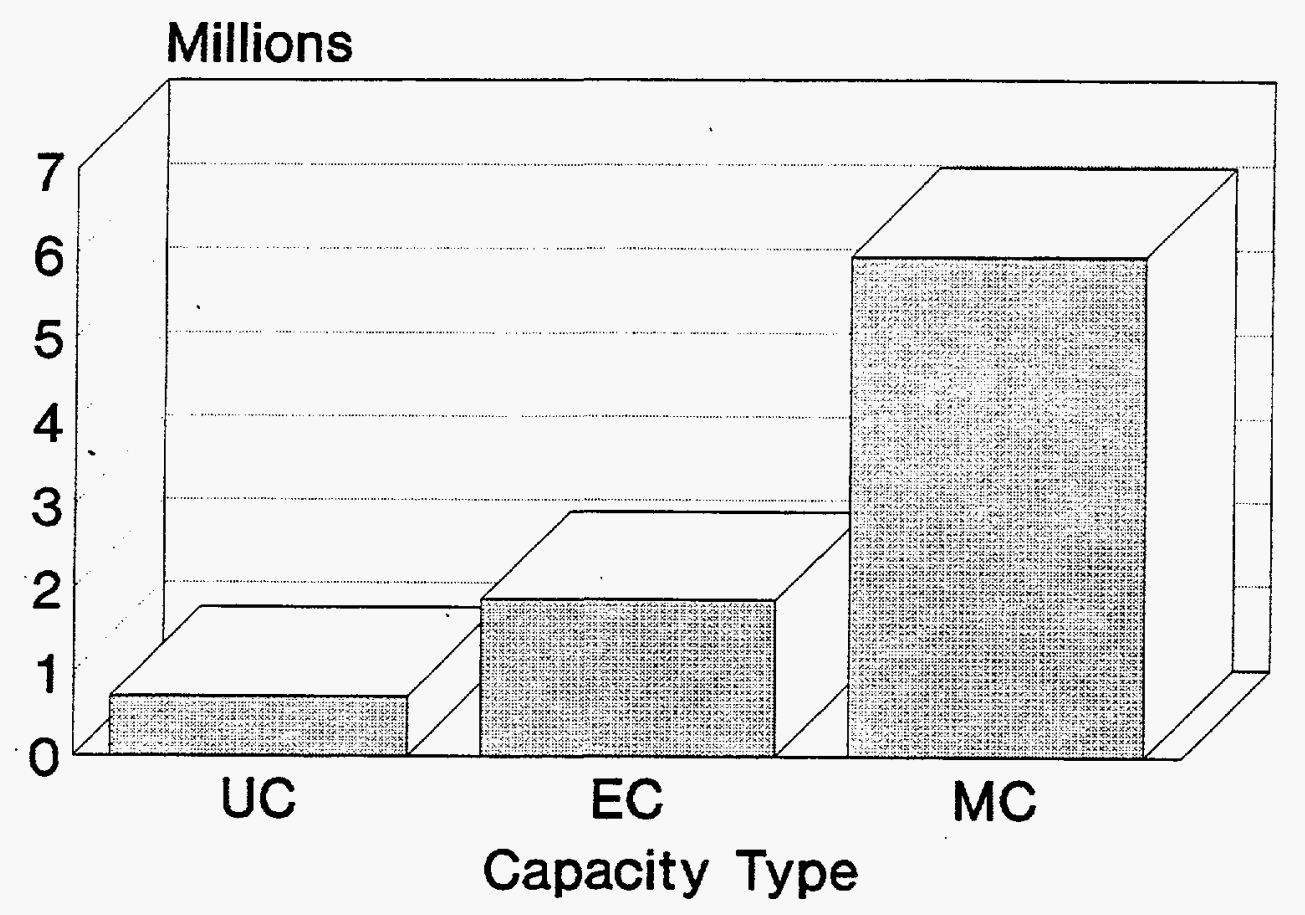

Figure 3: Total Annual Capacity in Sample Analyses by Capacity Type

Figure 4 presents distribution of the analytical capacities among Operations Offices, and these are further broken down into the individual laboratories in Figure 5. Based on the current UC values, the majority of the DOE analytical capacity is being utilized at the Oak Ridge Operations Office, in particular at the laboratories at K-25, Y-12, Portsmouth, and Paducah. This predominance of capacity at Oak Ridge is not, however, so distinct for the EC and MC values where laboratories at Hanford, Rocky Flats, and Savannah River form large fractions of the DOE capacities. This is particularly clear in the case of the Hanford-222S laboratory, for example. Its share of overall DOE analytical capacity rises from just $5 \%$ for UC to $34 \%$ for EC and is at $24 \%$ for MC. These results seem to show that at Hanford $222 \mathrm{~S}$ in particular, and also at some of the other major laboratories, that while capacity is available, it is not presently being as effectively utilized there as at other sites.

Distribution of the annual capacities between the radiation control zones is shown in Table III. It is immediately clear that most utilized analytical capacity in the DOE Complex is in the RO and R1 categories, those RCZs in which the lowest radiation protection, if any, is required. Only about $3 \%$ of the overall utilized DOE capacity is utilized in shielded hoods, gloveboxes, or hot cells. The existing capacity for handling and analyzing high rad level samples is, however, about an order of magnitude higher than the capacity that is currently being utilized. Therefore, if sample loads of high 


\section{a) UC}

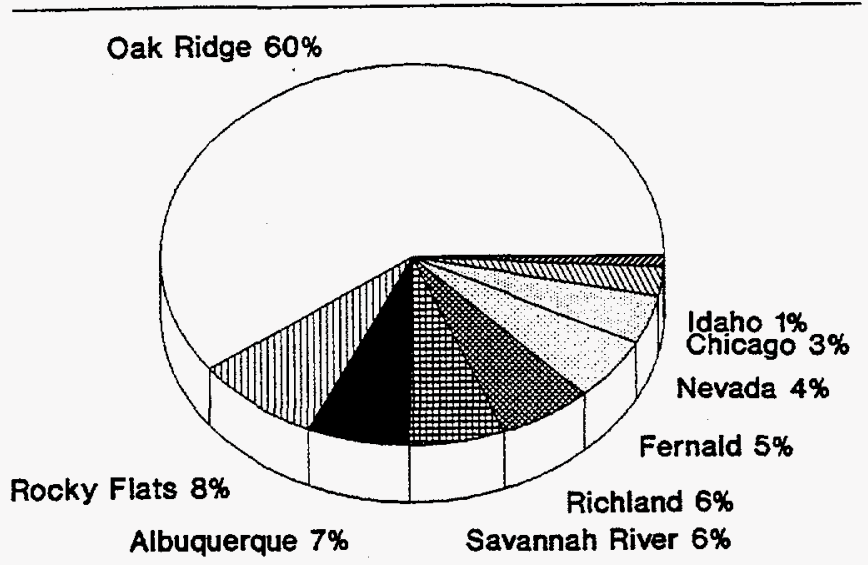

b) $\mathrm{EC}$

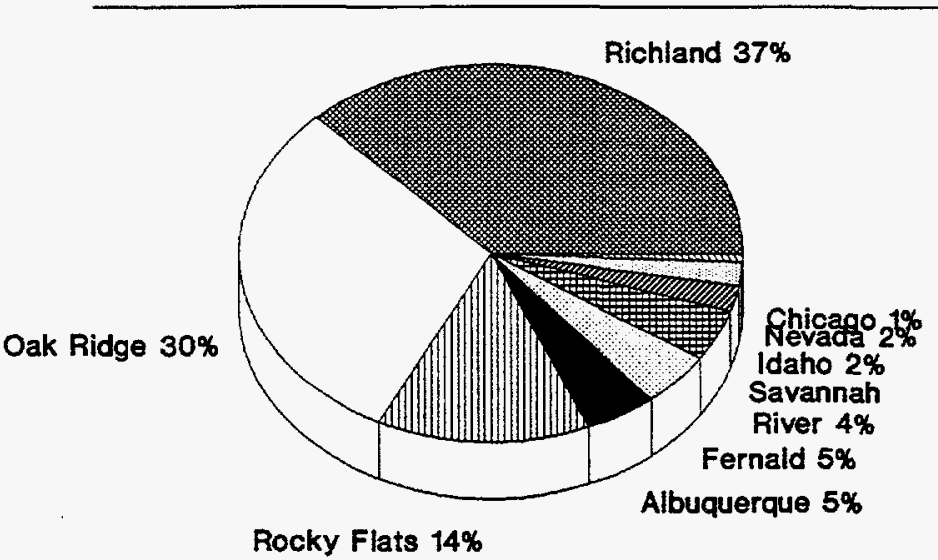

c) $\mathrm{MC}$

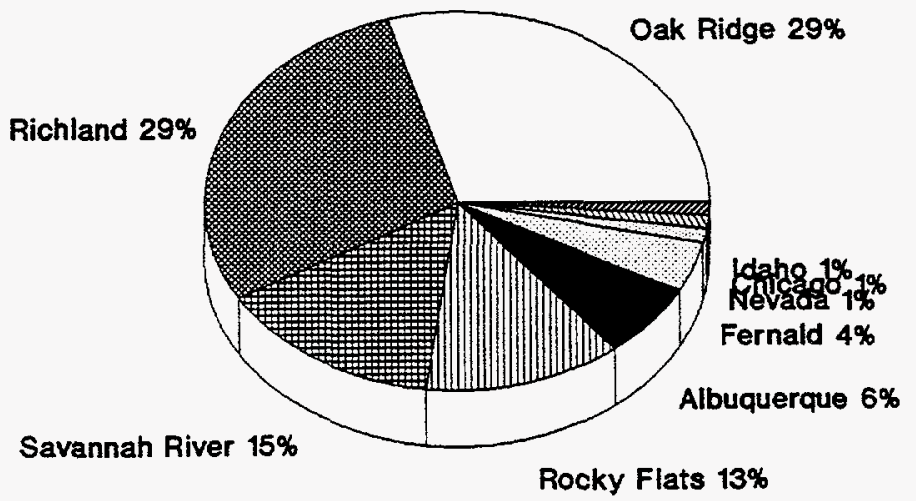

Figure 4: Distribution of Sample Analyses by Operations Office 


\section{a) UC}

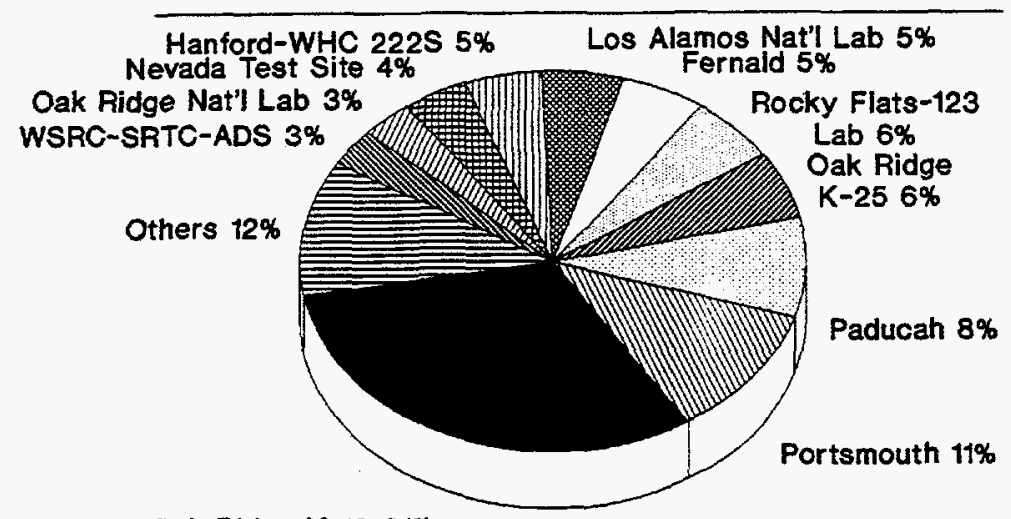

Oak Ridge $Y-1231 \%$

\section{b) EC}

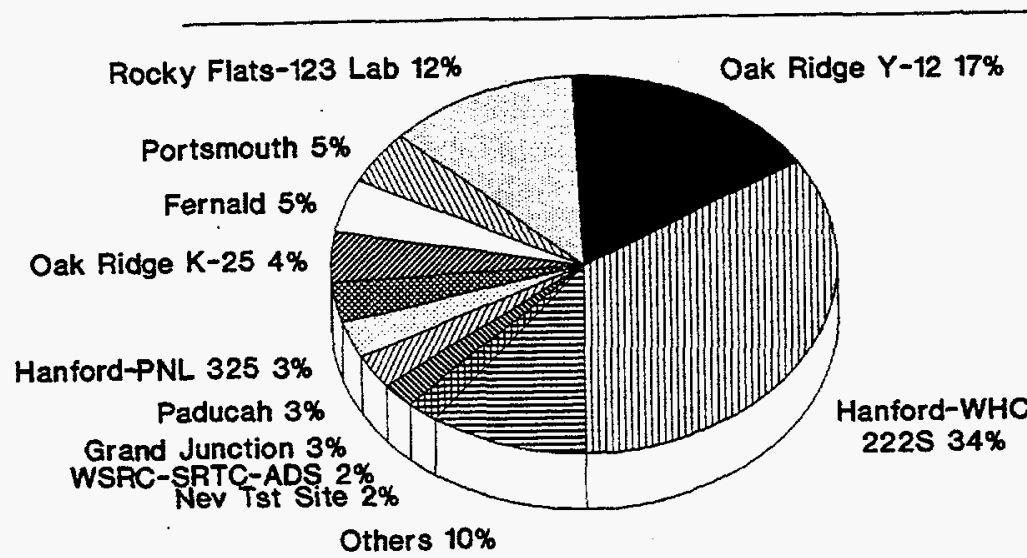

c) MC

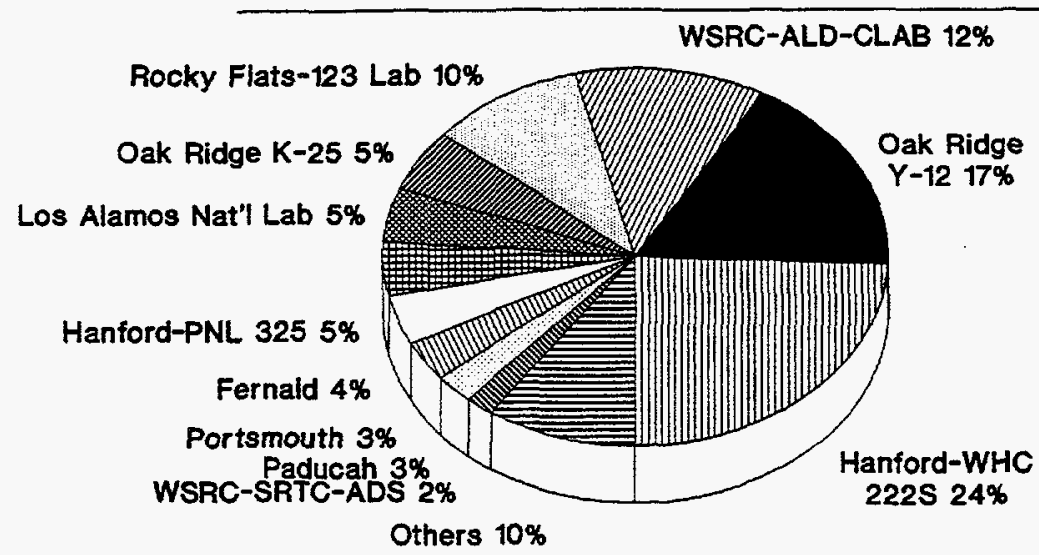

Others 10\%

Figure 5: Distribution of Sample Analyses by Laboratory 
rad level samples do increase, there is currently sufficient capacity to handle increases up to an order of magnitude higher. The increase in capacity that could become available by upgrading the laboratories is fairly uniform for all the RCZs, with the exception of R4, where upgrading laboratories with additional hot cells could potentially increase existing capacity by a factor of about 8 .

Table III: Distribution of Sample Analyses (in thousands) by RCZ

\begin{tabular}{|c|c|c|c|}
\hline RCZ/Capacity & UC & EC & MC \\
\hline \hline RO & 196 & 471 & 1,490 \\
\hline R0/R1 & 234 & 574 & 1,430 \\
\hline R1 & 255 & 357 & 1,660 \\
\hline R2 & 15 & 431 & 1,210 \\
\hline R3 & 6 & 23 & 102 \\
\hline R4 & 1 & 10 & 76 \\
\hline
\end{tabular}

In Table IV, similar data are presented for the distribution of capacity with sample matrix type. Increases in capacity from UC to EC and from EC to MC are found to be almost independent of the matrix type. This is not unexpected, since much analytical instrumentation, and hence capacity, may be used for all matrix types. Differences with matrix type would only be expected to be observed in the capacity to perform analytical sample preparation laboratory operations.

Table IV: Distribution of Sample Analyses (in thousands) by Matrix Type

\begin{tabular}{|c|r|r|r||}
\hline $\begin{array}{c}\text { Matrix/ } \\
\text { Capacity }\end{array}$ & UC & EC & MC \\
\hline \hline M1 & 371 & 1,081 & 3,525 \\
\hline M2 & 274 & 454 & 1,490 \\
\hline M3 & 62 & 331 & 957 \\
\hline
\end{tabular}

A key factor affecting laboratory capacity is the regulatory protocol that the sample analyses should follow. This can affect the operating times and number of quality control samples and calibration cycles that are required in completing a sample 
analysis. Figure 6 shows distributions of sample analyses among the analytical protocols for UC, EC, and MC. $83 \%$ of the utilized capacity is currently used to perform sample analyses according to regulatory protocols, while the remainder is support to production, waste treatment, or other processes. Participants in the study estimated that if their laboratories performed at their existing capacity, the fraction of sample analyses performed according to regulatory protocols will decrease to $71 \%$, and would further decrease to $65 \%$ at their maximum capacity.

Currently, just over half the utilized analytical capacity of the DOE Complex consists of sample analyses funded by EM, as seen in Figure 7. With the expected increase in EM activities at DOE sites, the participants in the study estimated that the fraction of EM-funded sample analyses would increase to between 70 and $80 \%$ if the laboratories performed at their existing or upgradable capacities. It is apparent, then, that the laboratories expect that any increase in sample loads at their laboratories will be coming mainly from EM-funded projects.

In order to plan any changes in DOE laboratory capacity, it is also necessary to determine individual sample analysis capacities in order to identify how the analytical resources may be more effectively utilized. Figures 8,9 , and 10 present data on UC, $E C$, and $M C$ respectively, showing the distribution of DOE analytical capacity by sample analysis type.

Over half the sample analyses within UC are radiochemical sample analyses, and inorganic sample analyses are about one third of the total. Most of the radiochemical sample analyses are gross alpha/beta measurements that have relatively short operating times, and most of the inorganic sample analyses are inductively coupled plasma-atomic emission spectroscopy (ICP-AES) and graphite furnace-atomic absorption (GF-AA) metals analyses. Organics constitute about $8 \%$ of $U C$ and are mainly volatile organic analysis-gas chromatography/massspectrometry (VOA-GC/MS) or analyses for pesticides, herbicides, and polychlorinated biphenyls (PCBs). Similar patterns of distribution of sample analyses are observed for the EC and MC data, except that there exists a large additional capacity for performing other organic sample analyses.

\subsection{Conclusions}

This study represents the first attempt at estimating DOE analytical laboratory capacity that is available or potentially available to EM. A model was developed and demonstrated at almost all the major DOE analytical laboratories for collecting analytical capacity data Complex-wide. The reliability of the results compiled from the data collected were found to be reasonable. The model was applied not only for collecting data on the laboratory capacity currently being utilized, but was also extended to give information on existing capacity available but not presently being utilized, and on the capacity that could be achieved by upgrading the laboratories. It was found that while much available capacity that is not being utilized exists for sample analyses performed in the R2, R3, and R4 RCZs, it is being used with about equal effectiveness across most matrix and sample analysis types. The effectiveness 
a) UC

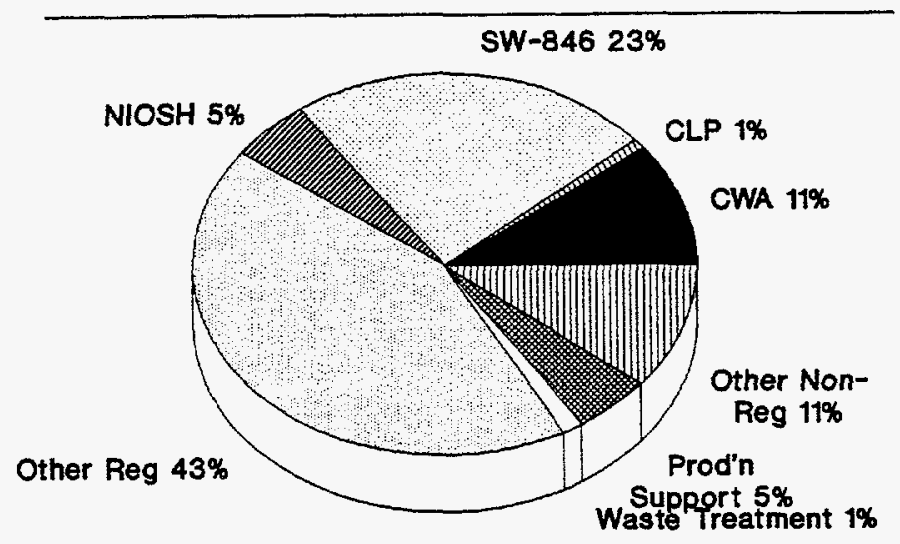

b) $\mathrm{EC}$

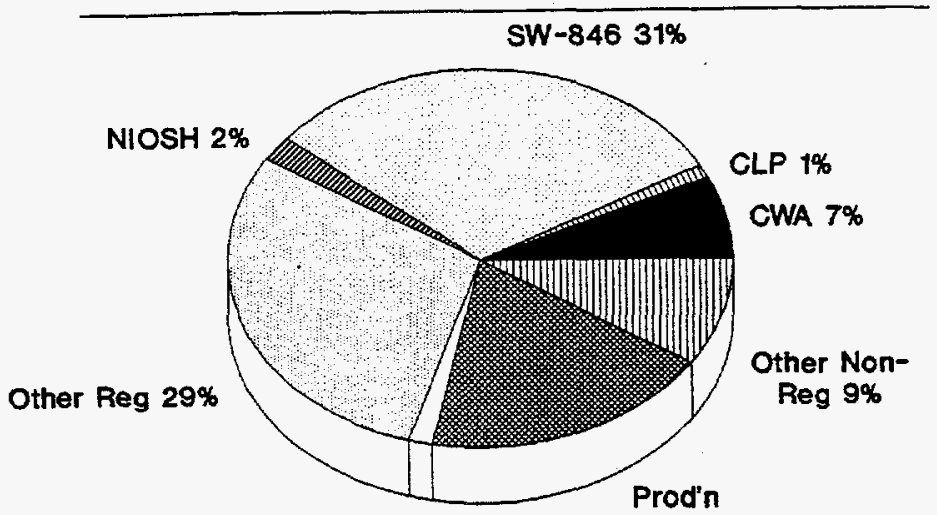

Waste Treatment 1\%

Support $19 \%$

c) $\mathrm{MC}$

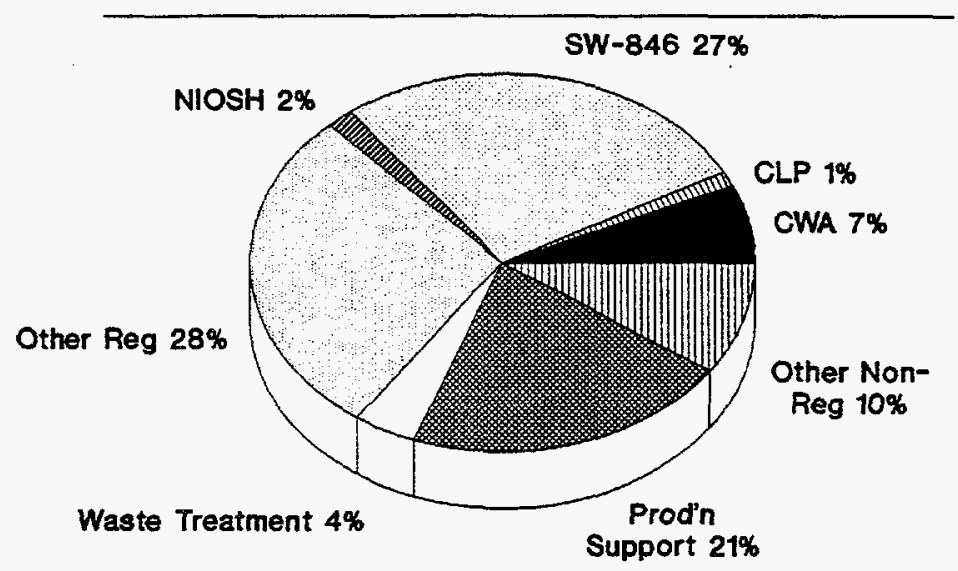

Figure 6: Distribution of Sample Analyses by Analytical Protocol 
a) UC

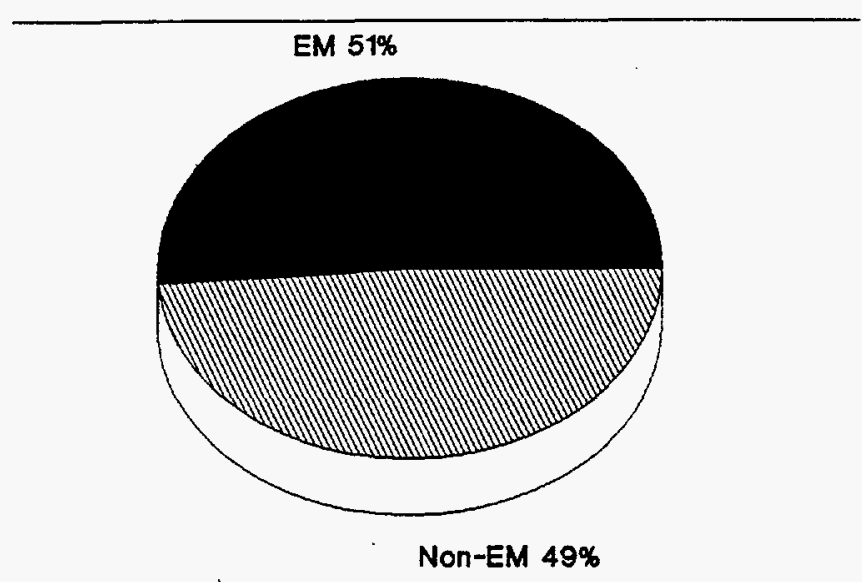

b) EC

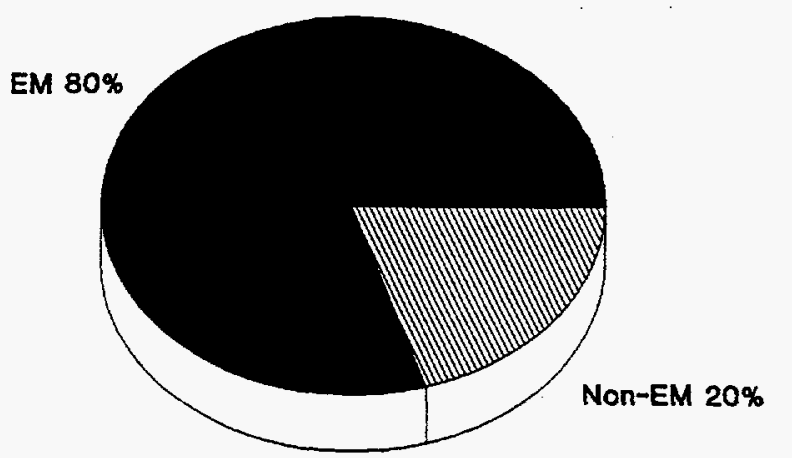

c) $\mathrm{MC}$

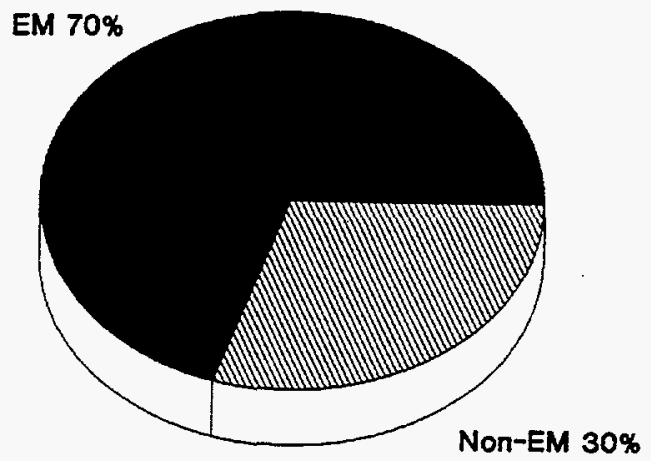

Figure 7: Distribution of Sample Analyses by Funding Source 


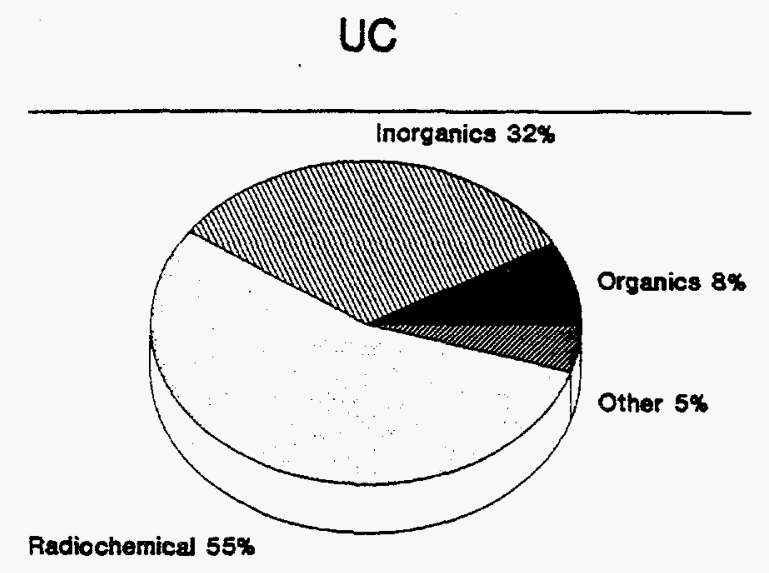

a) Organics

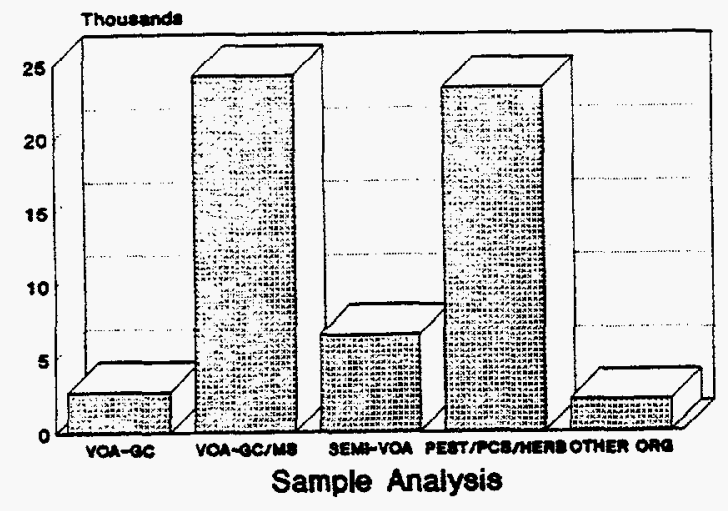

c) Radiochemical

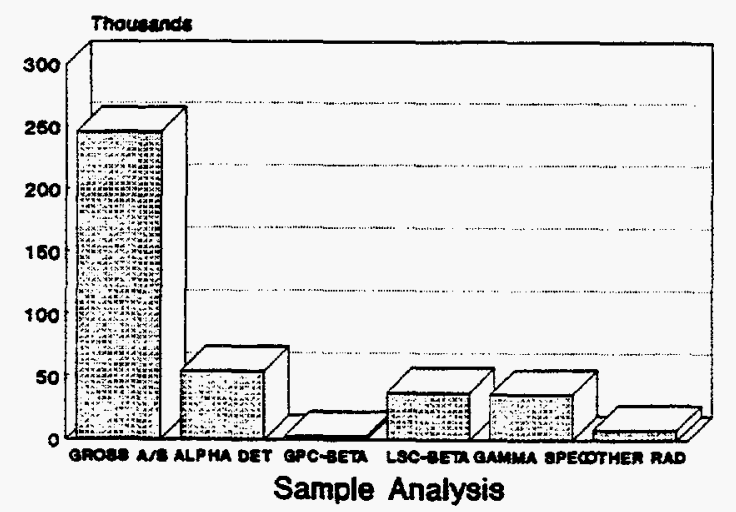

b) Inorganics

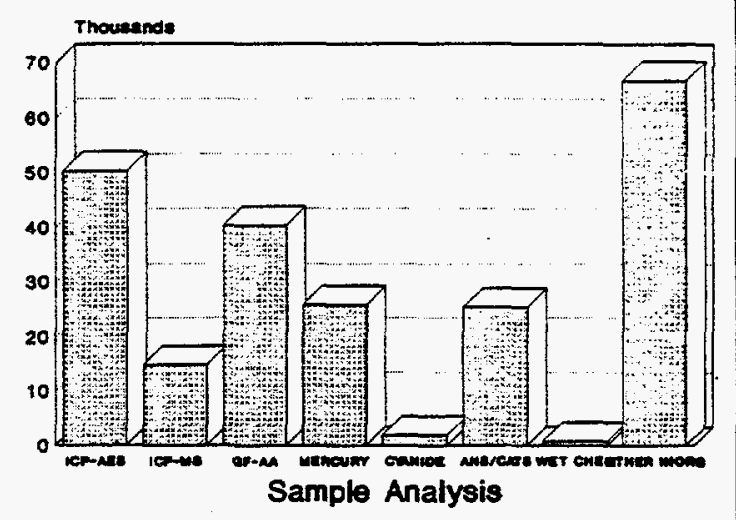

d) Other

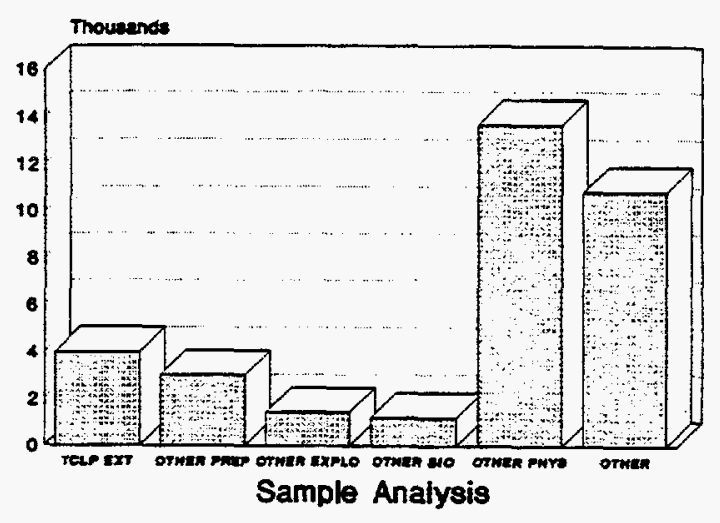

Figure 8: Distribution of Utilized Capacity by Sample Analysis Type 
EC

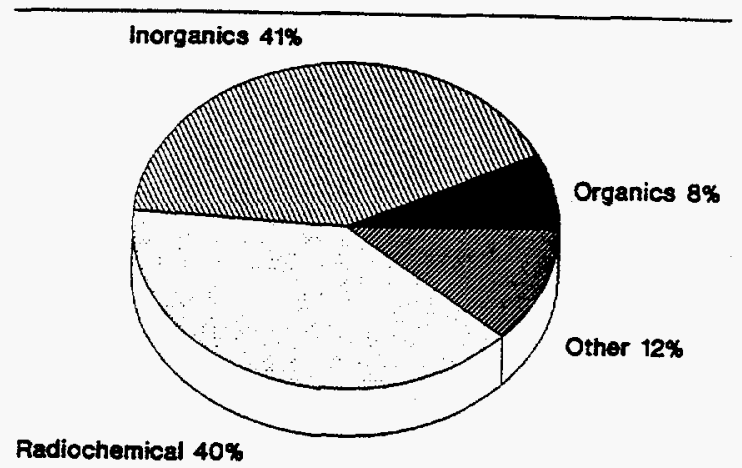

a) Organics

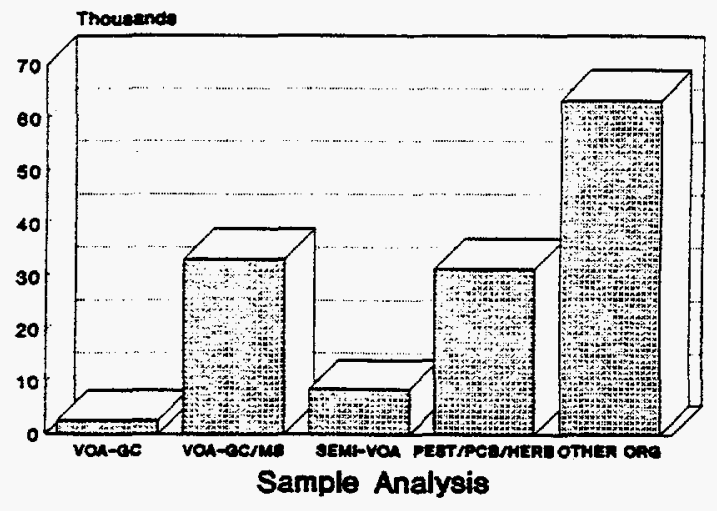

c) Radiochemical

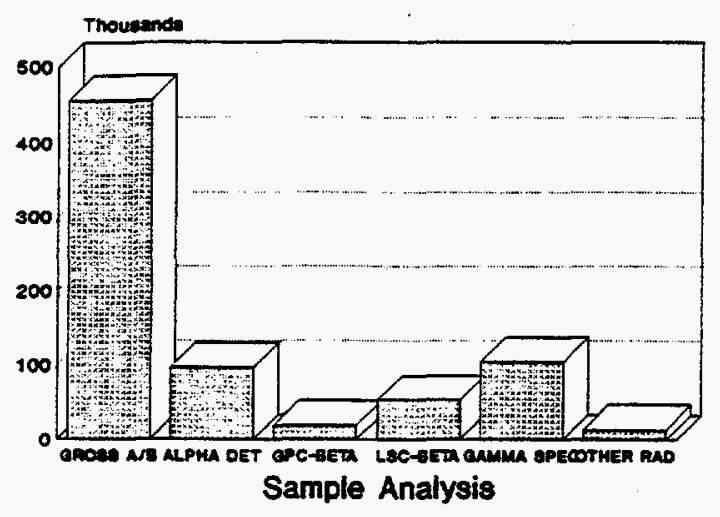

b) Inorganics

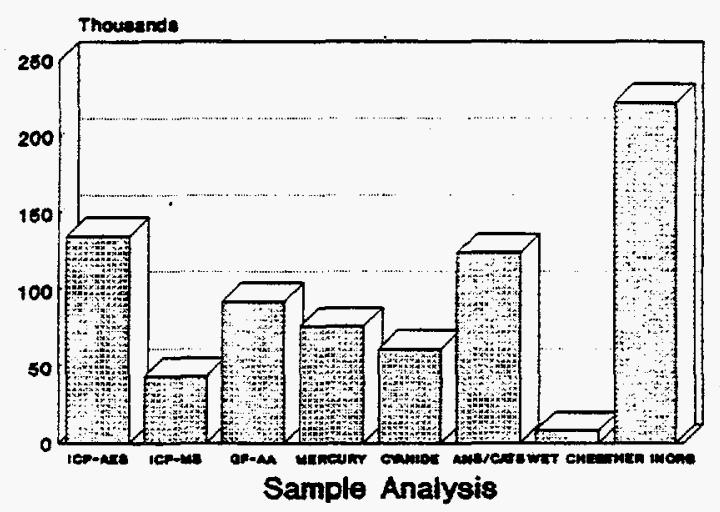

d) Other

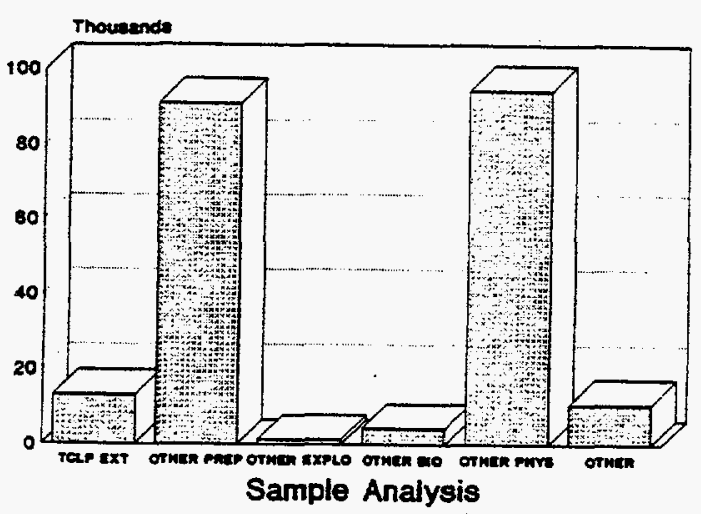

Figure 9: Distribution of Existing Capacity by Sample Analysis Type 


\section{MC}

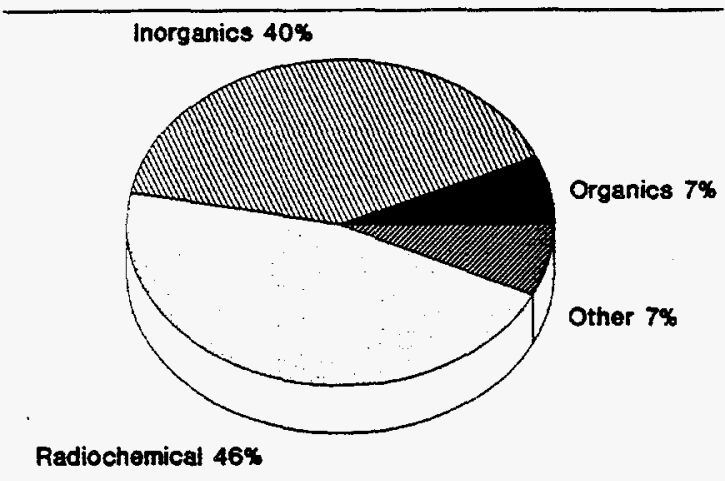

a) Organics

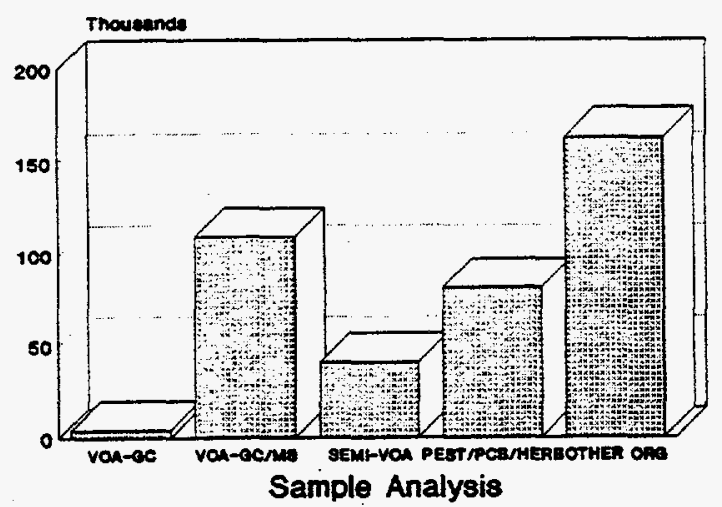

c) Radiochemical

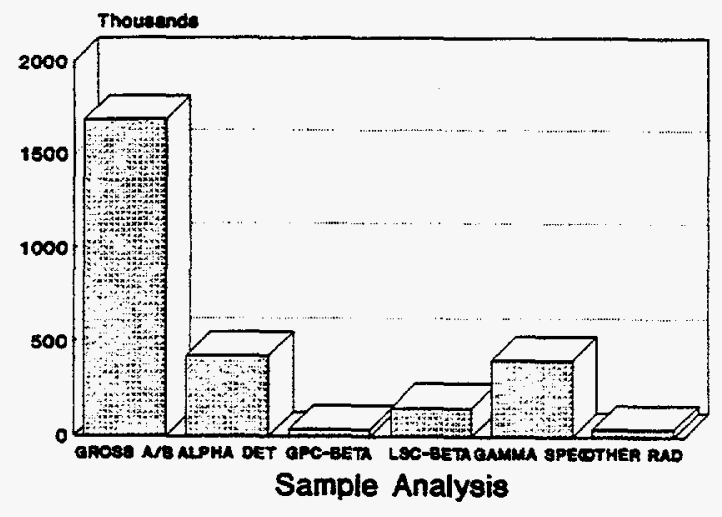

b) Inorganics

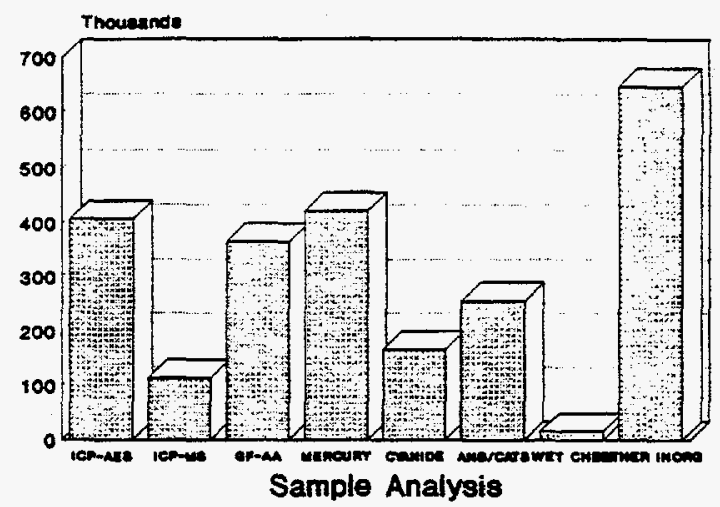

d) Other

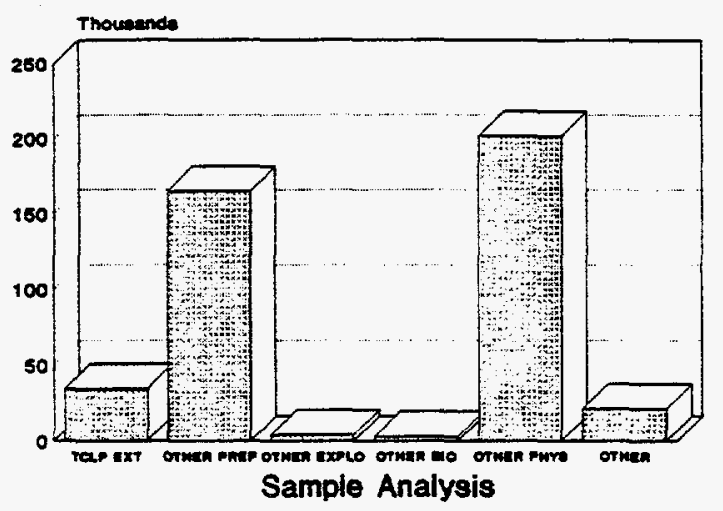

Figure 10: Distribution of Upgradable Capacity by Sample Analysis Type 
of the use of the capacity also varies between laboratories. Some laboratories have much higher factors of increase in going from UC to EC and $M C$ than others.

The data collected in this study therefore provide not only a picture of the analytical capabilities and capacity within the DOE Complex, but also may be used to compare with EM analytical needs and to form the basis for making major decisions on laboratory planning. 


\subsection{Introduction}

The EM Analytical Needs Assessment was carried out to provide EM-263 with a scenario of EM sampling and analysis needs until FY 99. Sampling and analysis needs arise from many EM activities, and accurate projections of these needs will become a key planning tool for EM in making critical management decisions on the allocation of analytical resources.

A model was developed in which data on current EM sampling and analysis needs is extrapolated to future years based on planned EM activities and budgets. This model improves upon previous efforts to assess EM analytical needs, ${ }^{1,2}$ since it clearly defines terms, relies on historical data to give a limit on the reliability of the projections, and uses overall EM planning documents and budgets to ensure complete coverage of the DOE Complex.

\subsection{Model Description}

The EM analytical needs assessment model is based on refinement of earlier models developed by EM-263, piloted at Hanford, and applied across the DOE Complex in FY 92. ${ }^{2}$ The essential steps taken in applying the model are presented below:

- The following information is collected:

- Descriptions of activities performed and planned under each EM ADS at major DOE installations;

- Historical data on actual numbers of environmental samples collected and sample analyses performed in FY 93 for each activity; and

- $\quad$ EM budget data for past, current and future fiscal years.

- Sample and sample analysis profiles are created for each EM activity based on the FY 93 sampling and analysis data supplied by the major DOE sites.

- Projections of the numbers of environmental samples to be collected and sample analyses to be performed at the major DOE sites are made for the period FY 94 - FY 99 based on planned EM activities.

- Projections of total EM sampling and analysis needs across the DOE Complex for the period FY 94 - FY 99 are made by extrapolating the projections for the major sites using the EM budget figures.

This process is shown schematically in Figure 11.

As with the analytical laboratory capacity model, the units used for expressing EM analytical needs are sample analysis cycles. This allows comparison of the data generated by the two models (See Section 4.0). However, the lists of permitted sample analysis types for the two models are not identical, since the capacity data are 


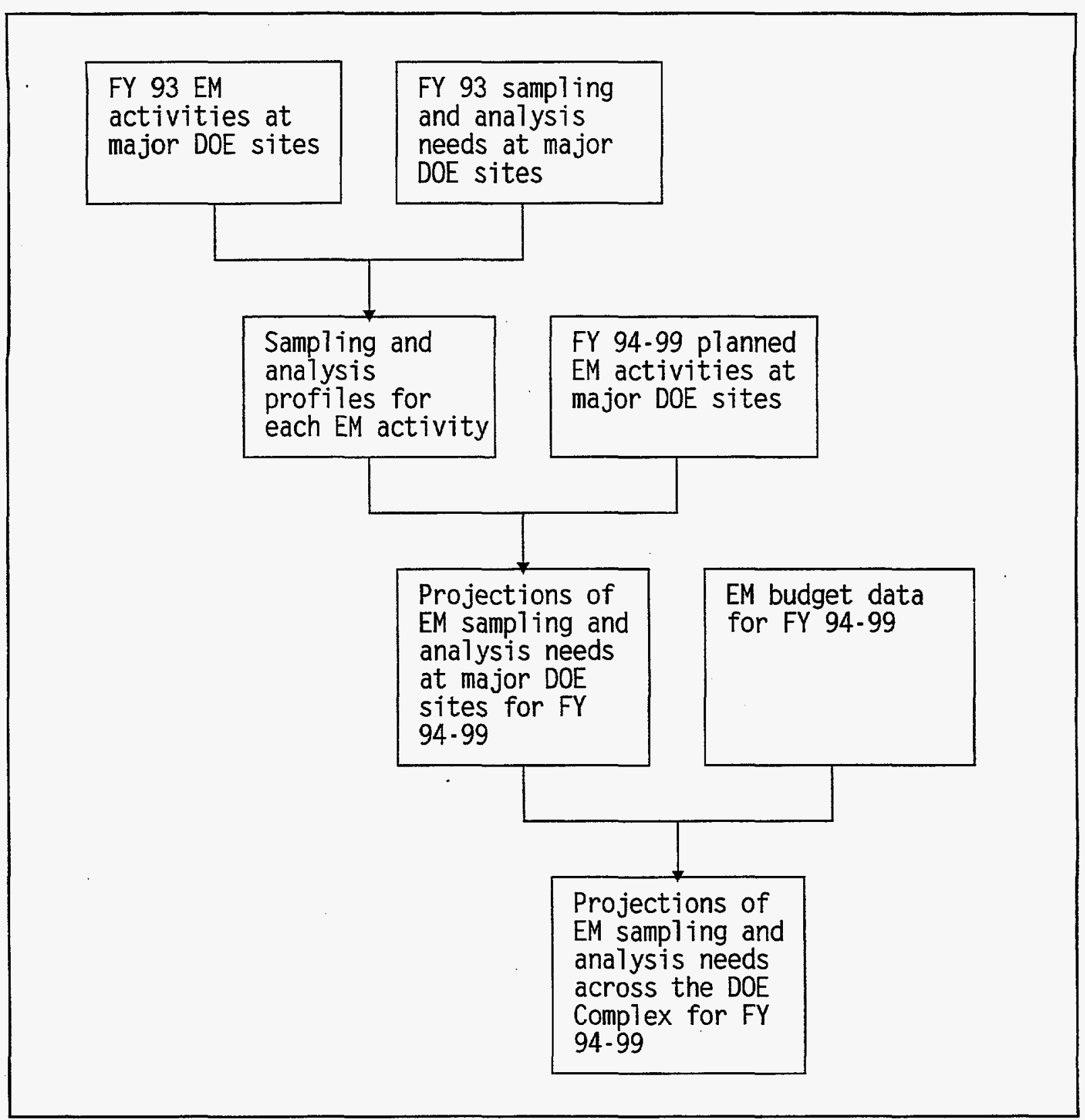

\section{Figure 11: EM-263 Analytical Needs Model}

derived from equipment usage in the laboratory, while the needs data are derived from analytical requests made by EM customers. This issue is also addressed in Section 4.0. The list of sample analyses used in the EM analytical needs model is given in Appendix E, and is the same as the sample analysis list used in the NSTS.

EM analytical needs are also categorized according to the activity level and matrix type of the samples in a similar fashion to the capacity model. While definitions for the three matrix types (M1-M3) are identical to those given in Section 2.2, additional estimates of sample activity are provided for RO-R4 categories in the needs model. These are shown below: 
RO - Non-radioactive

R1 - $<10 \mathrm{mR} / \mathrm{hr}$ on contact, $<100 \mathrm{nCi}$ alpha/g, or $<100 \mathrm{nCi}$ alpha/ml

R2 - $10-200 \mathrm{mR} / \mathrm{hr}$ on contact, $<100 \mathrm{nCi}$ alpha/g, or $<100 \mathrm{nCi}$ alpha/ml

R3 - $<200 \mathrm{mR} / \mathrm{hr}$ on contact, $>100 \mathrm{nCi}$ alpha/g, or $>100 \mathrm{nCi}$ alpha/ml

R4 - $>200 \mathrm{mR} / \mathrm{hr}$ on contact

The EM cleanup work performed in FY 93 and planned for FY $94-99$ is also categorized according to a specific set of activities. The list of permitted activities is presented in Appendix E.

\subsection{Model Implementation}

The model was implemented in the following three stages:

1) Needs data collection from major DOE sites;

2) Data review and entry into the model; and

3) Complex-wide summarization of the data.

\subsubsection{Data Collection}

Instructions for completing the reporting forms for the needs model were distributed to the DOE sites listed in Table V. Sites were requested to provide a report summarizing their EM activities requiring sampling and analysis in hard copy. A final report containing detailed data on FY 93-99 activity and the analytical needs for FY 93 was requested for electronic submission on Lotus $1-2-3^{\circledR}$ spreadsheets. A copy of the reporting formats and instructions package sent to the participants is provided in Appendix E.

Table V: Participation of DOE Sites in the EM-263 Needs Assessment

\begin{tabular}{|l|l|c|l|}
\hline \multicolumn{1}{|c|}{ Ops Office } & \multicolumn{1}{|c|}{ Site } & $\begin{array}{c}\text { Data } \\
\text { Received }\end{array}$ & Comments \\
\hline \hline ALBUQUERQUE & Los Alamos Nat'I Lab & $N$ & No data received \\
\hline FERNALD & Fernald & $Y$ & \\
\hline IDAHO & Idaho Nat'I Eng Lab & $Y$ & \\
\hline NEVADA & Nevada Test Site & $Y$ & \\
\hline OAKRIDGE & Oak Ridge - K25 & $Y$ & \\
\hline OAKRIDGE & Oak Ridge Nat'I Lab & $Y$ & \\
\hline OAKRIDGE & Oak Ridge - Y12 & $Y$ & \\
\hline RICHLAND & Hanford & Some & Minimal data received, not incorporated into model \\
\hline ROCKY FLATS & Rocky Flats & $Y$ & Data is incomplete \\
\hline SAVANNAHRIVER & Savannah River & $Y$ & No data received for EM-40 activities \\
\hline
\end{tabular}

All EM ADSs from the ten major DOE installations were reviewed by EM-263 to determine whether sampling and analysis is required within each ADS. Sites were then provided with tables prepared by EM-263 containing its assessment of their sampling and analysis needs. The sites were further requested to confirm EM-263's 
assessment and to provide summary information on the activities performed under each ADS requiring sampling and analysis.

The sites were then asked to provide the following more detailed information on each ADS activity requiring environmental sampling and analysis in a Lotus 1-2-3 spreadsheet:

- A general description of the activity, including its regulatory driver, the number of units addressed, the activity schedule, and activity costs;

- Activity sampling and analysis information, including the schedule, average batch size, total numbers of samples and sample analyses performed and planned in the activity, and for FY 93, the numbers of customer samples taken and sample analyses requested categorized by radiation level, matrix type, and sample analysis type;

- Names and references of planning documents, project reports, budgetary documents, and regulatory documents associated with the activity.

Needs data were requested from the ten DOE sites with the largest fraction of the EM budget. Data were received from nine of the sites, although the data were not always complete or useable. Data received from Savannah River represented only EM-30 analytical needs, and data received from Hanford were of insufficient detail to be entered in the model. No data were received from Los Alamos National Laboratory.

\subsubsection{Data Review}

Each set of hardcopy data received from the field was reviewed for consistency and completeness. Any inconsistent, blank, or apparently incorrect data were questioned through telephone conversations with the field point of contact. In a number of cases, sites were only able to provide a limited amount of the requested data, and best estimates of the planned activities were made. Therefore, the needs data used for compiling this report were the best available obtained from the sites.

\subsubsection{Data Summarization}

The best available needs data received from the laboratories were summarized using a set of Lotus 1-2-3 ${ }^{\circledR}$ spreadsheets. Macros in the spreadsheets were used to generate a series of database files with consistent site, ADS number, activity, and regulatory driver names, a series of database files containing the number of unit-years of an activity per fiscal year, and activity sampling and analysis profiles. An example of a sampling and analysis profile is given in Figure 12. An additional set of macros was used to generate a set of tables summarizing EM sampling and analysis needs. A detailed description of the data summarization spreadsheet system is given in Appendix F, and the summarized tables of needs data are given in Appendix G. All results presented in the following section of the report was abstracted from data collected in Appendix G. 


\section{ACTIVITY SAMPLING AND ANALYSIS PROFLE (NSTS/NEEDS)}

FOR ONE UNTT - YEAR

\begin{tabular}{|ll|}
\hline Activity: & RI/FS \\
\hline Unit: & OU \\
\hline Reguletory Driver: & \multicolumn{1}{|c|}{0} \\
\hline Filename: & SUM2NEED.WK3 \\
\hline Date: & September 21.1994 \\
\hline
\end{tabular}

\begin{tabular}{|l|c|}
\hline Average S\&A Duration per Unit (yrs): \\
\hline Total Samples/Unit-yr: & 224.1 \\
\hline Total Samp Ans/Unit-yr: & 2020.9 \\
\hline Average Batch Size: & \\
\hline \# of Unit-yrs in FY 93: & 16.4 \\
\hline
\end{tabular}

\begin{tabular}{|c|c|c|c|c|c|c|c|c|c|}
\hline & Ro & R1 & $\mathrm{R2}$ & R3 & R4 & M1 & M2 & $\mathrm{M3}$ & $\mathrm{C}$ \\
\hline SAMPLES TAKEN & & & & $\because$ & & & & & E \\
\hline CUSTOMER SAMPLES & 67.9 & 201.1 & 15.2 & 0.01 & 0.0 & 51.5 & 108.8 & 3.8 & 0.0 \\
\hline SAMPLE ANALYSES REQUESTED & & & & & & & & 26 & \\
\hline ORGANICS & & $\cdots$ & & & 3 & & & & 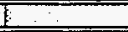 \\
\hline Volatiles by GC/MS & 95.7 & 31.4 & 3.7 & 0.0 & 0.0 & 52.1. & 77.0 & 1.7 & 0.0 \\
\hline Volatiles by GC & 20.2 & 0.9 & 0.0 & 0.0 & 0.0 & 0.0 & 21.0 & 0.8 & 0.0 \\
\hline Semi-Volaties & 51.6 & 28.1 & 3.7 & 0.0 & 0.0 & 32.3 & 49.8 & 0.9 & 0.0 \\
\hline Herbicides/Pesticides/PCBs & 57.9 & 35.7 & 0.0 & 0.0 & 0.0 & 21.8 & 71.8 & 0.0 & 0.0 \\
\hline Other Organics & 0.0 & 6.5 & 0.0 & 0.0 & 0.0 & 5.7 & 0.8 & 0.0 & 0.0 \\
\hline INORGANICS & & & & & & & & & \\
\hline RCRA Appendix IX Matals & 0.0 & 1.3 & 0.0 & 0.0 & 0.0 & 0.0 & 1.3 & 0.0 & 0.0 \\
\hline CAM (California) Metals (RCRA) & 0.0 & 0.0 & 0.0 & 0.0 & 0.0 & 0.0 & 0.0 & 0.0 & 0.0 \\
\hline RCRA TCLP Metals and EPTOX Mel & 0.0 & 4.9 & 0.0 & 0.0 & 0.0 & 0.1 & 4.8 & 0.0 & 0.0 \\
\hline EPA CLP Metals (CERCLA) & 102.7 & 56.8 & 10.0 & 0.0 & 0.0 & 40.6 & 124.9 & 4.1 & 0.0 \\
\hline NPDES Metals (Clean Water Act) & 24.5 & 0.0 & 0.0 & 0.0 & 0.0 & 24.5 & 0.0 & 0.0 & 0.0 \\
\hline Drinking Water Metals (SDWA) & 0.0 & 0.0 & 0.0 & 0.0 & 0.0 & 0.0 & 0.0 & 0.0 & 0.0 \\
\hline Metals by Other Reguletory Suite & 78.4 & 0.0 & 0.0 & 0.0 & 0.0 & 25.8 & 55.4 & 2.2 & 0.0 \\
\hline Metals by Suite, Other & 0.0 & 46.7 & 1.9 & 0.0 & 0.0 & 28.1 & 15.5 & 0.0 & 0.0 \\
\hline Metals by Metal Except Mercury & 2.5 & 8.6 & 0.0 & 0.0 & 0.0 & 1.6 & 9.6 & 0.0 & 0.0 \\
\hline Mercury & 24.5 & 7.4 & 0.0 & 0.0 & 0.0 & 24.5 & 7.3 & 0.0 & 0.0 \\
\hline Anions/Cations - IC & 36.7 & 18.5 & 0.0 & 0.0 & 0.0 & 53.7 & 1.5 & 0.0 & 0.0 \\
\hline Inorganics Wet Chemistry & 104.3 & 31.7 & 0.0 & 0.0 & 0.0 & 83.5 & 52.5 & 0.0 & 0.0 \\
\hline Other Inorganics & 30.9 & 9.6 & 0.0 & 0.0 & 0.0 & 29.7 & 10.8 & 0.0 & 0.0 \\
\hline RADIOACTIVES & & $\ldots$ & & & 3 & & & & \\
\hline Gross Alpha/Beta & 197.8 & 63.8 & 2.7 & 0.0 & 0.0 & 90.9 & 168.0 & 5.4 & 0.0 \\
\hline Gamma Scan & 69.6 & 20.1 & 1.5 & 0.0 & 0.0 & 33.0 & 54.1 & 4.1 & 0.0 \\
\hline Transuranic & 0.0 & 0.0 & 0.0 & 0.0 & 0.0 & 0.0 & 0.0 & 0.0 & 0.0 \\
\hline Plutonium isotopes & 71.5 & 15.0 & 1.0 & 0.0 & 0.0 & 21.5 & 64.7 & 1.3 & 0.0 \\
\hline Uranium Isotopes & 90.0 & 43.2 & 1.0 & 0.0 & 0.0 & 37.2 & 95.7 & 1.3 & 0.0 \\
\hline Curium Isotopes & 0.0 & 1.8 & 0.0 & 0.0 & 0.0 & 0.9 & 0.9 & 0.0 & 0.0 \\
\hline Thorium Isotopes & 0.0 & 9.7 & 1.0 & 0.0 & 0.0 & 2.7 & 8.0 & 0.0 & 0.0 \\
\hline Radium Isotopes & 23.8 & 0.0 & 0.0 & 0.0 & 0.0 & 16.4 & 7.4 & 0.0 & 0.0 \\
\hline Americium-241 & 71.1 & 5.4 & 1.0 & 0.0 & 0.0 & 21.4 & 54.8 & 1.3 & 0.0 \\
\hline Neptunium-237 & 0.0 & 6.8 & 0.0 & 0.0 & 0.0 & 0.7 & 6.2 & 0.0 & 0.0 \\
\hline Polonium-210 & 0.0 & 0.0 & 0.0 & 0.0 & 0.0 & 0.0 & 0.0 & 0.0 & 0.0 \\
\hline Tritium & 45.3 & 33.4 & 4.9 & 0.0 & 0.0 & 34,6 & 48.1 & 0.9 & 0.0 \\
\hline Radon-222 & 0.0 & 0.0 & 0.0 & 0.0 & 0.0 & 0.0 & 0.0 & 0.0 & 0.0 \\
\hline Lead-210 & 0.0 & 0.0 & 0.0 & 0.0 & 0.0 & 0.0 & 0.0 & 0.0 & 0.0 \\
\hline Cerbon-14 & 0.0 & 0.0 & 0.0 & 0.0 & 0.0 & 0.0 & 0.0 & 0.0 & 0.0 \\
\hline Nickei-63 & 0.0 & 0.0 & 0.0 & 0.0 & 0.0 & 0.0 & 0.0 & 0.0 & 0.0 \\
\hline Selenium-79 & 0.0 & 0.0 & 0.0 & 0.0 & 0.0 & 0.0 & 0.0 & 0.0 & 0.0 \\
\hline Technetium-99 & 0.0 & 1.2 & 0.3 & 0.0 & 0.0 & 0.3 & 1.2 & 0.0 & 0.0 \\
\hline Strontium isotopes & 0.0 & 0.0 & 0.0 & 0.0 & 0.0 & 0.0 & 0.0 & 0.0 & 0.0 \\
\hline Strontium-89 & 0.0 & 0.0 & 0.0 & 0.0 & 0.0 & 0.0 & 0.0 & 0.0 & 0.0 \\
\hline Strontium-90 & 0.0 & 15.0 & 0.3 & 0.0 & 0.0 & 1.5 & 13.9 & 0.0 & 0.0 \\
\hline lodine-129 & 0.0 & 0.0 & 0.0 & 0.0 & 0.0 & 0.0 & 0.0 & 0.0 & 0.0 \\
\hline Nickel-59 & 0.0 & 0.0 & 0.0 & 0.0 & 0.0 & 0.0 & 0.0 & 0.0 & 0.0 \\
\hline Total Urenium & 1.6 & 0.0 & 0.0 & 0.0 & 0.0 & 1.6 & 0.0 & 0.0 & 0.0 \\
\hline Total Thorium & 0.0 & 0.0 & 0.0 & 0.0 & 0.0 & 0.0 & 0.0 & 0.0 & 0.0 \\
\hline Total Strontium & 37.0 & 0.0 & 0.0 & 0.0 & 0.0 & 13.8 & 21.9 & 1.3 & 0.0 \\
\hline Other RAD & 105.4 & 26.7 & 3.0 & 0.0 & 0.0 & 10.6 & 124.6 & 0.0 & 0.0 \\
\hline OTHER & & & & & & & & & \\
\hline Other Wet Chemistry & 80.0 & 0.0 & 0.0 & 0.0 & 0.0 & 60.2 & 19.9 & 0.0 & 0.0 \\
\hline TCLP Extraction & 0.0 & 6.2 & 0.1 & 0.0 & 0.0 & 1.1 & 5.2 & 0.0 & 0.0 \\
\hline Asbestos & 0.0 & 6.4 & 0.0 & 0.0 & 0.0 & 0.0 & 6.4 & 0.0 & 0.0 \\
\hline Industrial Hygiene, NOS & 2.1 & 0.0 & 0.0 & 0.0 & 0.0 & 0.3 & 1.8 & 0.0 & 0.0 \\
\hline Physical Properties & 10.8 & 3.2 & 0.6 & 0.0 & 0.0 & 5.4 & 9.3 & 0.0 & 0.0 \\
\hline Other, Not Otherwise Specified (NO & 0.0 & 1.8 & 0.0 & 0.0 & 0.0 & 1.8 & 0.0 & 0.0 & 0.0 \\
\hline
\end{tabular}

\footnotetext{
Figure 12: Example of an Activity Sample and Analysis Profile
} 


\subsection{Results}

An overall summary of projected EM analytical demand is given in Table VI, which presents projections of the EM demand for sample analyses for each reporting site, as well as EM Office and Complex-wide totals extrapolated using EM budget figures for FY 93-FY 99. A similar summary of projected EM sample demand is given in Table VII.

Table VI: Total Projected EM Sample Analysis Needs (in thousands) by Fiscal Year and Site

\begin{tabular}{|c|c|c|c|c|c|c|c|}
\hline Fiscal Year & 93 & 94 & 95 & 96 & 97 & 98 & 99 \\
\hline \multicolumn{8}{|l|}{ Site } \\
\hline Fernald & 70 & 111 & 110 & 11 & 10 & 16 & 10 \\
\hline INEL & 34 & 36 & 41 & 39 & 35 & 40 & 40 \\
\hline Nevada Test Site & 1 & 31 & 48 & 49 & 49 & 49 & 48 \\
\hline Oak Ridge K-25 & 16 & 350 & 350 & 35 & 34 & 32 & 32 \\
\hline Oak Ridge $x-10$ & 15 & 24 & 22 & 16 & 16 & 15 & 15 \\
\hline Oak Ridge $Y-12$ & 4 & 5 & 5 & 5 & 4 & 4 & 3 \\
\hline Rocky Flats & 58 & 175 & 113 & 104 & 103 & 102 & 100 \\
\hline Savannah River & 67 & 69 & 69 & 69 & 70 & 70 & 70 \\
\hline Other Sites & 425 & 1429 & 1425 & 612 & 593 & 608 & 586 \\
\hline & & & & & & & \\
\hline \multicolumn{8}{|l|}{ EM Otfice } \\
\hline$E M-30$ & 264 & 325 & 323 & 293 & 298 & 292 & 286 \\
\hline$E M-40$ & 276 & 1757 & 1671 & 474 & 443 & 473 & 448 \\
\hline$E M-60$ & 113 & 143 & 126 & 126 & 127 & 127 & 127 \\
\hline \multicolumn{8}{|l|}{ Complex } \\
\hline TOTAL EM & 691 & 2232 & 2183 & 939 & 914 & 937 & 905 \\
\hline
\end{tabular}

Overall computed projections of annual EM sample analysis and sample needs for $F Y$ 93-99 are presented in Figure 13. The estimated EM sample analysis needs for FY 93 were estimated to be around 690,000 , and this was projected to peak to over two million in FY 94 and FY 95, before levelling off at about 900,000 per year for the next four fiscal years. This is attributed mainly to a rapid increase in sample analysis needs for EM-40 the Office of Environmental Restoration, as can be seen in Figure 14 where the share of sample analysis needs is seen to increase from 42 percent in FY 93 to 79 percent in FY 94 . This phenomenon probably reflects the end of the characterization phase that EM-40 is currently undergoing for all its sites. A large number of critical decisions based on analytical data concerning environmental cleanups are expected to be made in FY 95 and 96 , and this probably accounts for the increased sample analysis demand immediately preceding this time period.

It should also be noted that no factor was taken into account regarding delays in meeting planned milestones. In many cases, backlogs of work may be created beyond 
Table VII: Total Projected EM Sample Needs (in thousands) by Fiscal Year and Site

\begin{tabular}{|c|c|c|c|c|c|c|c|}
\hline Fiscal Year & 93 & 94 & 95 & 96 & 97 & 98 & 99 \\
\hline \multicolumn{8}{|l|}{ Site } \\
\hline Fernald & 8 & 13 & 14 & 2 & 2 & 3 & 2 \\
\hline INEL & 11 & 11 & 13 & 15 & 13 & 15 & 15 \\
\hline Nevada Test Site & 0 & 3 & 10 & 10 & 10 & 10 & 10 \\
\hline Oak Ridge K-25 & 1 & 28 & 28 & 10 & 10 & 10 & 10 \\
\hline Oak Ridge $X-10$ & 6 & 9 & 9 & 6 & 6 & 6 & 6 \\
\hline Oak Ridge $Y-12$ & 0 & 1 & 1 & 1 & 1 & 1 & 1 \\
\hline Rocky Flats & 2 & 14 & 8 & 8 & 8 & 8 & 7 \\
\hline Savannah River & 28 & 28 & 28 & 28 & 28 & 28 & 28 \\
\hline Other Sites & 90 & 193 & 209 & 148 & 143 & 148 & 144 \\
\hline & & & & & & & \\
\hline \multicolumn{8}{|l|}{ EM Office } \\
\hline$E M-30$ & 98 & 108 & 108 & 106 & 108 & 108 & 105 \\
\hline$E M-40$ & 42 & 187 & 199 & 109 & 100 & 108 & 106 \\
\hline$E M-60$ & 3 & 3 & 3 & 3 & 3 & 3 & 3 \\
\hline & & & & & & & \\
\hline \multicolumn{8}{|l|}{ Complex } \\
\hline TOTAL EM & 146 & 301 & 321 & 228 & 220 & 228 & 223 \\
\hline
\end{tabular}

the planning term, and this may cause the peak in projected EM sampling and analysis requirements to be less pronounced and to be smoothed out over a longer period of time. Any correction of this type can only be made after additional application of the model, and will be included in updates to the needs data.

Of the sites that reported, there were considerable fluctuations in projected sample analysis demand. During the peak fiscal years of FY 94 and FY 95, the Oak Ridge K25 site appears to have the largest analytical demand, although this drops by a factor of 10 in the following years. Other sites, such as INEL and Savannah River, are projected to have a much more constant demand over the period FY 93 through FY 99. Note, however, that Savannah River only reported data for activities funded by EM-30, which corresponds to the majority of work performed on the site, and not for activities funded by EM-40. Figure 15 shows the distribution of projected sample analysis needs by site across the Complex. It may be seen that the reporting sites only represent under 40 percent of the total EM budget, and there is considerable fluctuation in the projected sample analysis demand between these sites.

Projections were also made regarding the number of customer samples that need to be taken for the projected analytical demand. The annual change in projected sample count is also presented in Figure 13, and it essentially follows the same trends observed for sample analyses. A maximum of around 320,000 samples is projected for FY 95 before it levels at around 220,000 samples.

Annual projected EM analytical needs for various radiation levels of samples are shown in Table VIII. In general, EM analytical demand decreases with increasing radiation level of samples. Exceptions occur, however, during the projected peak years of FY 94 and FY 95, when demand for analyses of samples at the R1 and R2 radiation levels is expected to increase by a much larger factor than for samples at the 


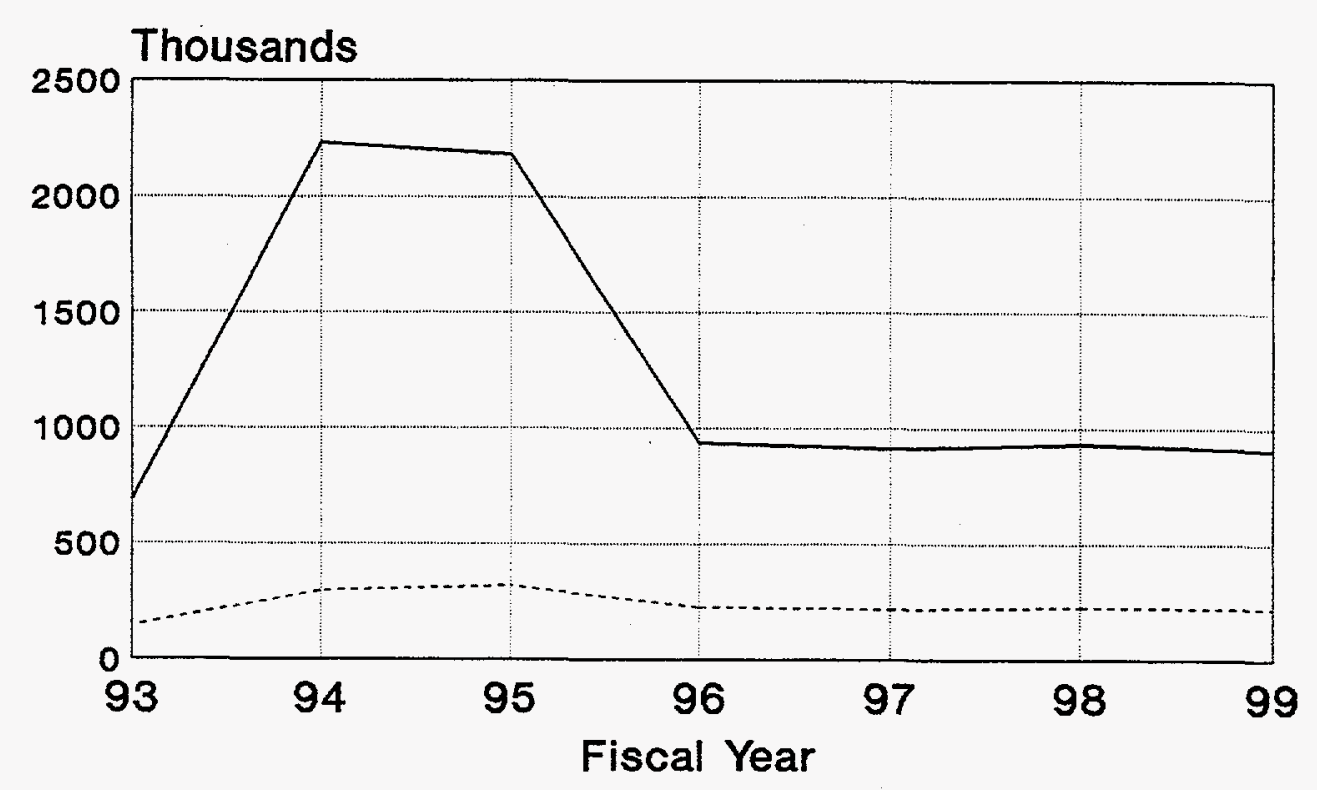

a) Sample Analyses

b) Samples

Figure 13: Total Projected Annual EM Sample and Sample Analysis Needs for FY 93-99

other radiation levels. This leads to a higher demand for analyses of R1 samples than for analyses of RO samples in FY 94 and FY 95.

Table VIII: Distribution of EM Sample Analysis Needs (in thousands) by Radiation Level

\begin{tabular}{|c|r|r|r|r|r|r|r||}
\hline Rad Level/FY & \multicolumn{1}{|c|}{93} & \multicolumn{1}{|c|}{94} & 95 & 96 & 97 & 98 & 99 \\
\hline \hline RO & 434 & 815 & 704 & 514 & 500 & 509 & 493 \\
\hline R1 & 275 & 1220 & 1276 & 436 & 423 & 438 & 428 \\
\hline R2 & 18 & 226 & 231 & 14 & 14 & 14 & 13 \\
\hline R3 & 3 & 10 & 14 & 14 & 16 & 16 & 11 \\
\hline R4 & 4 & 4 & 4 & 4 & 4 & 4 & 4 \\
\hline
\end{tabular}




\section{a) FY 93}

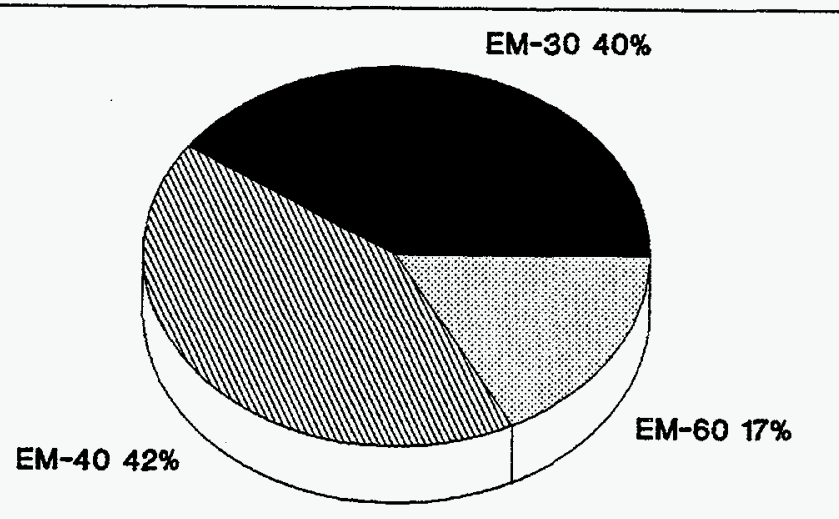

b) FY 94

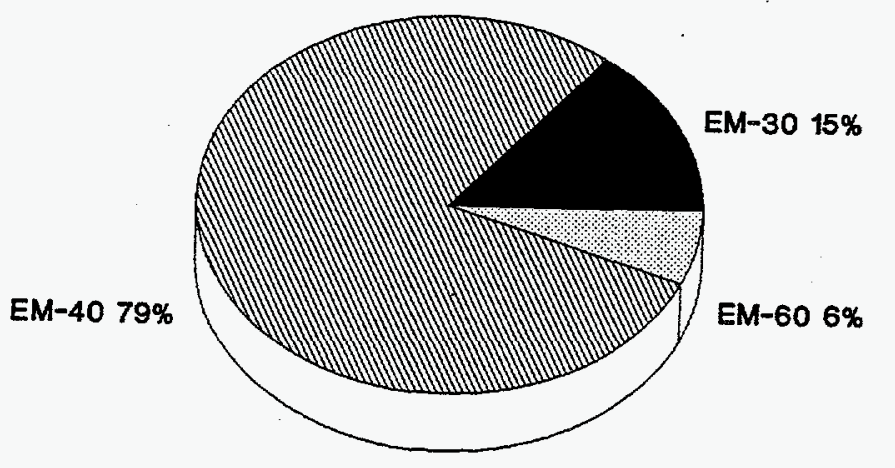

c) FY 95-99

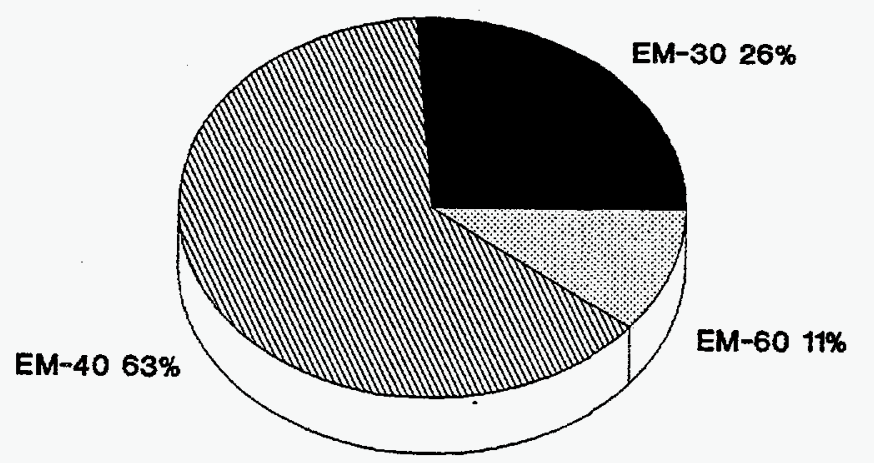

Figure 14: Distribution of Sample Analysis Needs by EM Office 


\section{a) FY 93}

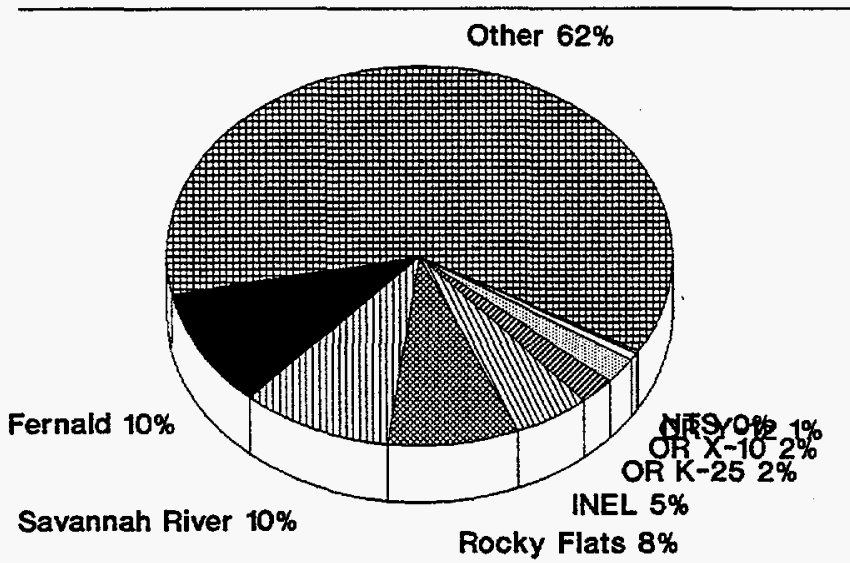

b) FY 94

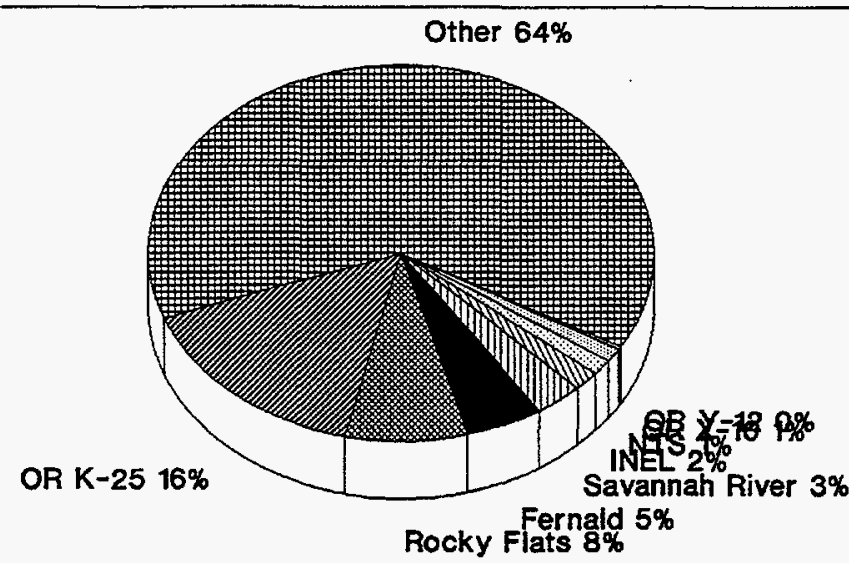

c) FY 95-99

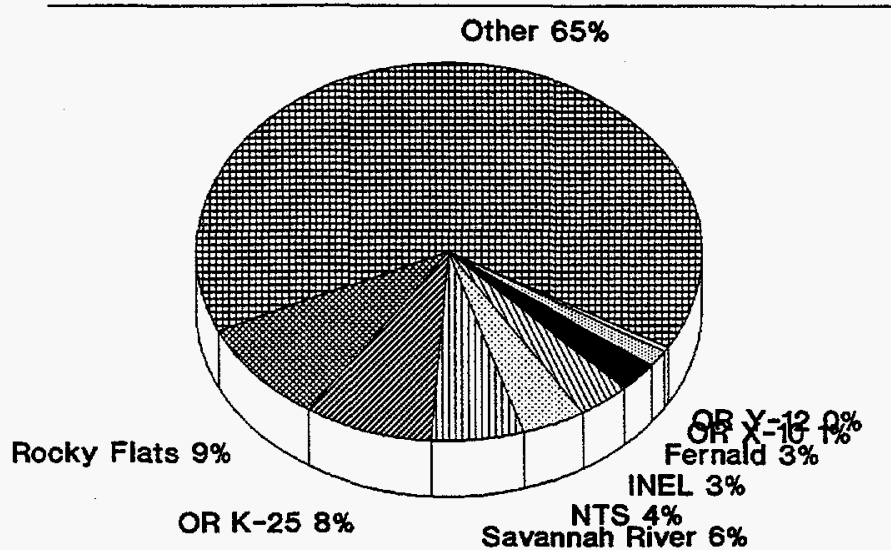

Figure 15: Distribution of Sample Analysis Needs by Site 
In Table IX, similar data are presented for the distribution of EM analytical demand with sample matrix type. In general, a little under half of the needed sample analyses are projected to be on samples of the simplest matrix type (M1), with the remainder being split somewhat in favor of M2 samples over M3 samples. This distribution over the three matrix type appears to be fairly consistent over the whole projection period.

Table IX: Distribution of EM Sample Analysis Needs (in thousands) by Matrix Type

\begin{tabular}{|c|r|r|r|r|r|r|r||}
\hline Matrix/FY & \multicolumn{1}{|c|}{93} & \multicolumn{1}{|c|}{94} & \multicolumn{1}{c|}{95} & \multicolumn{1}{c|}{96} & \multicolumn{1}{c|}{97} & \multicolumn{1}{c|}{98} & \multicolumn{1}{c|}{99} \\
\hline \hline M1 & 340 & 959 & 918 & 426 & 415 & 413 & 408 \\
\hline M2 & 186 & 640 & 598 & 367 & 352 & 366 & 357 \\
\hline M3 & 124 & 589 & 622 & 103 & 103 & 114 & 96 \\
\hline
\end{tabular}

Figure 16 presents the projected EM analytical demand distributed according to EM activity. The striking feature in this figure is the large fraction of projected sample analysis needs for hazardous waste management. This represents over 40 percent of total projected needs in FY 94, although this drops to 18 percent for the period FY 95-FY 99. The remaining sample analysis needs are distributed rather uniformly across the various cleanup activities.

The regulatory drivers of the activities generating the analytical demand determine the protocol used in performing the sample analyses. Figure 17 presents the distribution of sample analysis demand by regulatory-driven protocol. The largest fraction of EM sample analyses are driven by Resource Conservation and Recovery Act (RCRA) requirements, followed by Comprhensive Environmental Response, Compensation, and Liability Act (CERCLA) and state requirements, although a large number of sample analyses that follow other regulatory drivers that were not specified. The fraction of RCRA-driven sample analyses increases to its maximum in FY 94, suggesting that the large surge in sample analysis demand projected in FY 94 and FY 95 is primarily due to an increase in sample analyses from RCRA-driven activities.

EM sample analysis needs were also studied according to sample analysis type. This information is important in identifying the analytical resources required to meet the analytical demand. Figures 18,19 , and 20 present data on projected EM analytical demand for FY 93, FY 94, and FY 95 respectively, showing the distribution by sample analysis type.

Whereas the absolute numbers of sample analyses projected from year to year may vary considerably, the distribution of sample analyses by sample analysis type remains very constant. It is projected that 41-44 percent of EM analytical demand will be for radiochemical sample analyses, 30-34 percent for inorganic sample analyses, and 1314 percent for organic sample analyses. Even within each sample analysis class, the 
a) FY 93

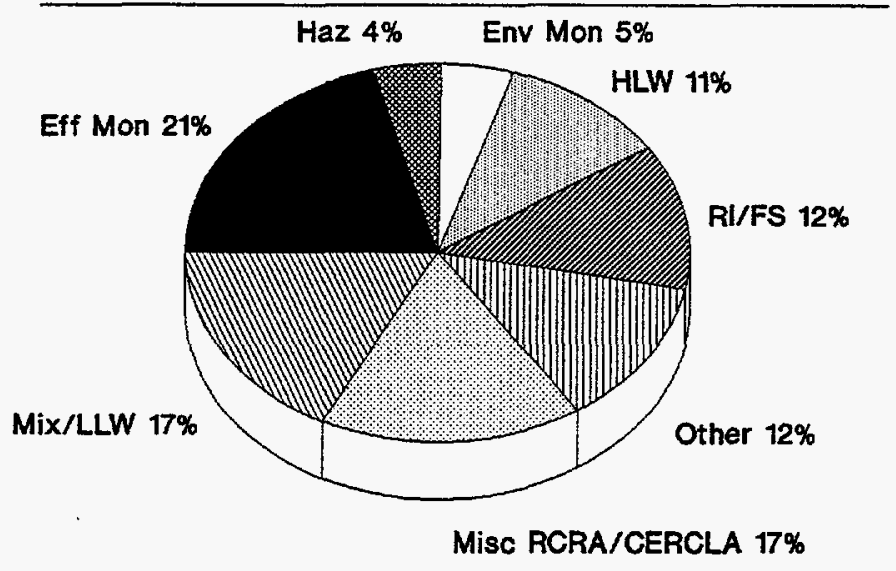

b) FY 94
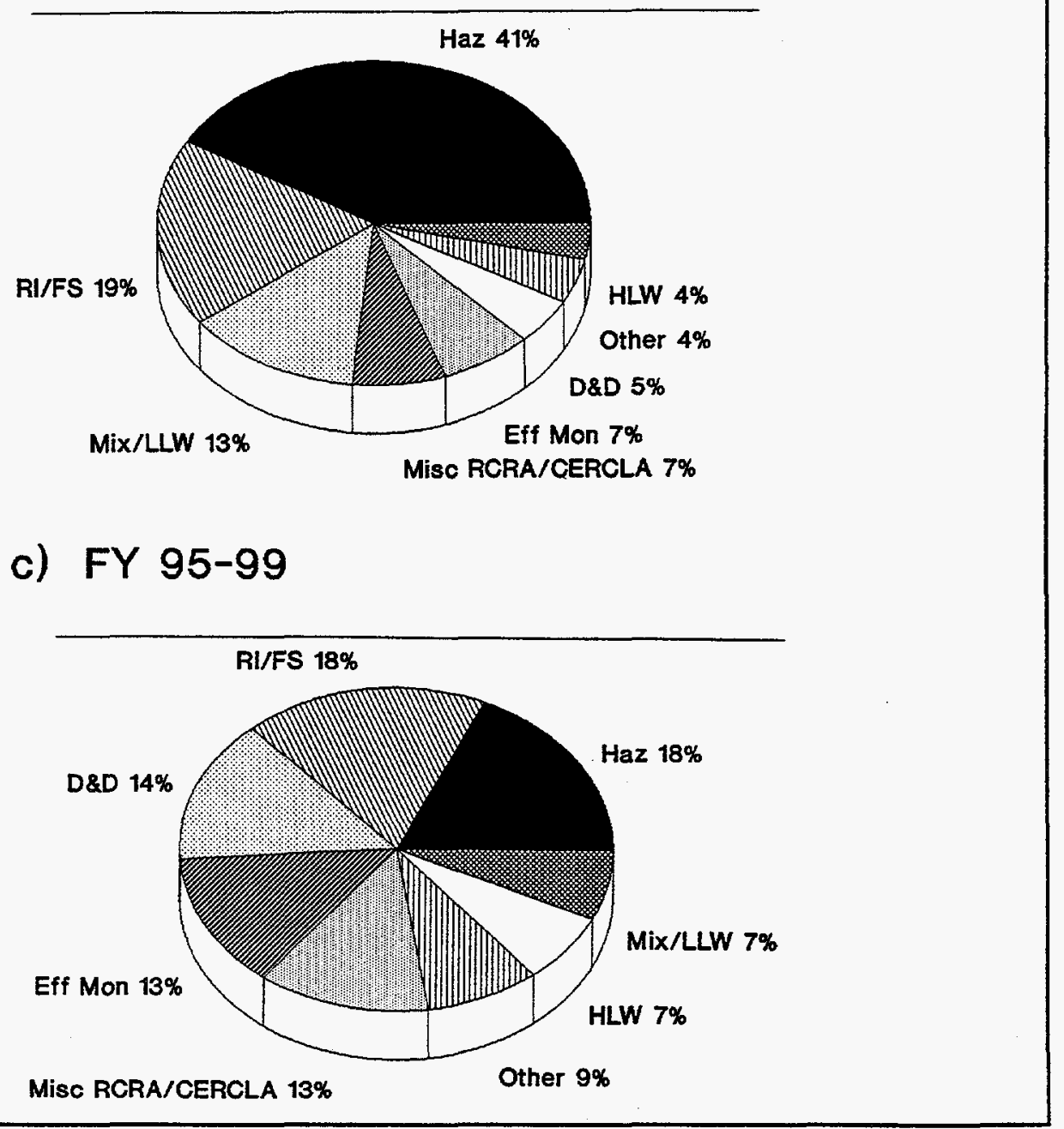

Figure 16: Distribution of Sample Analysis Needs by Activity 


\section{a) FY 93}

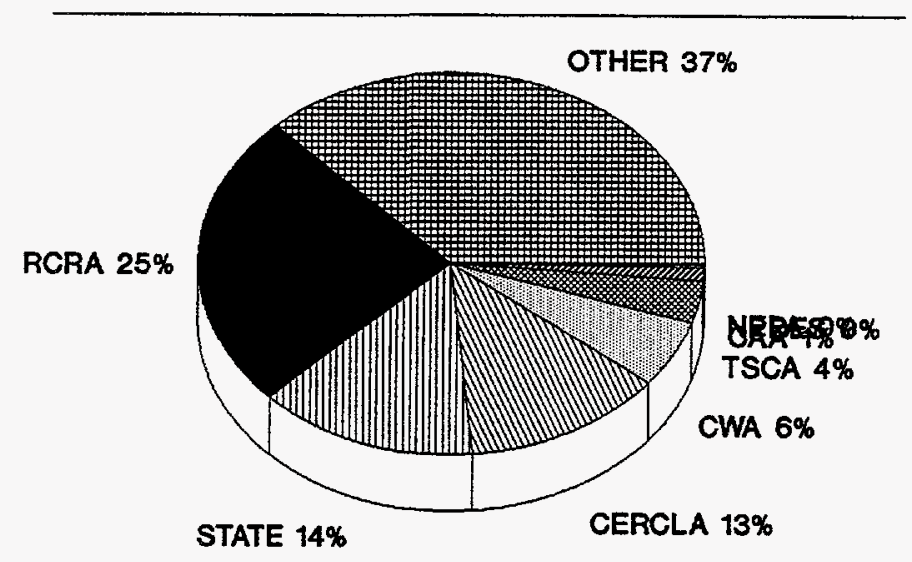

b) FY 94
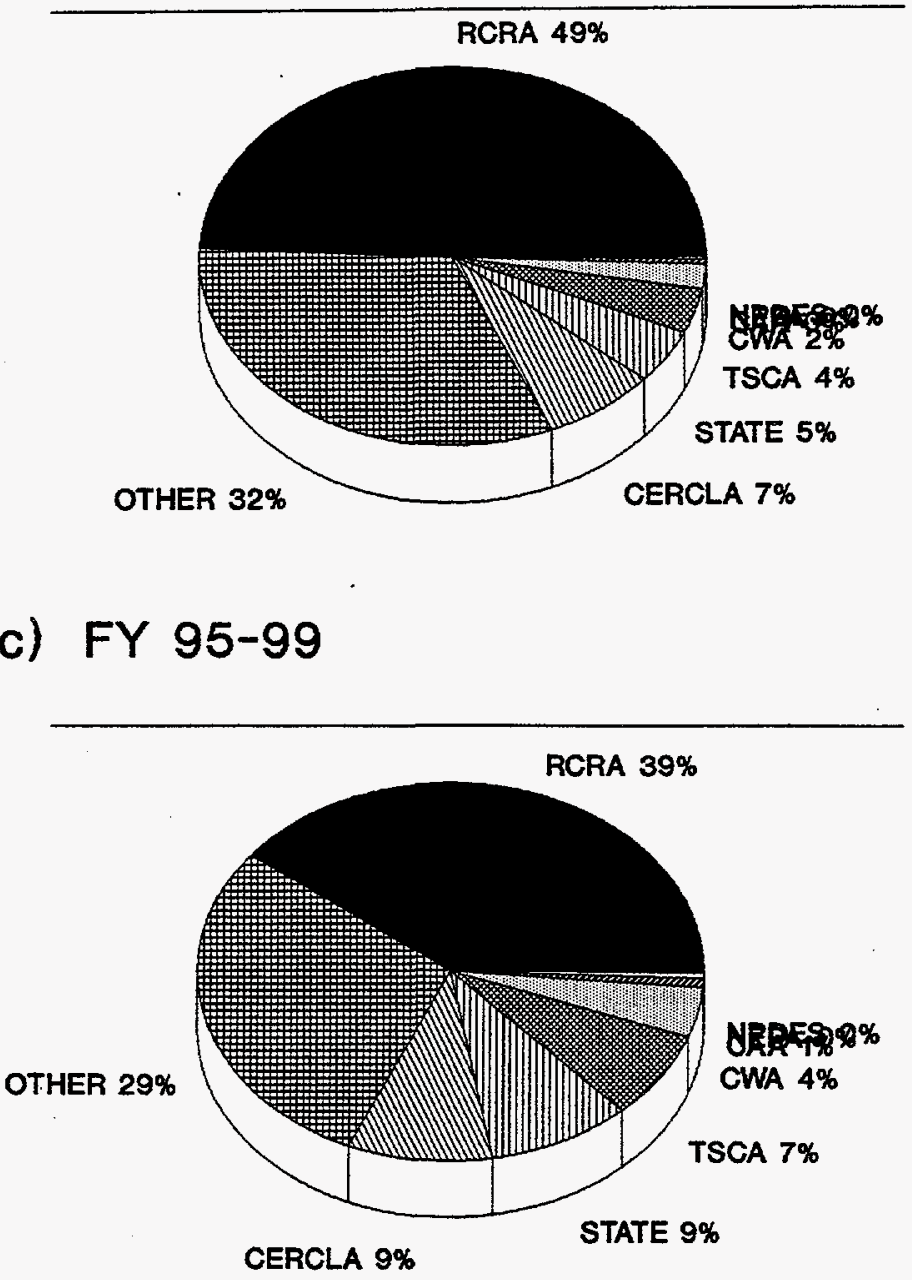

Figure 17: Distribution of Sample Analysis Needs by Regulatory Driver 


\section{FY 93}

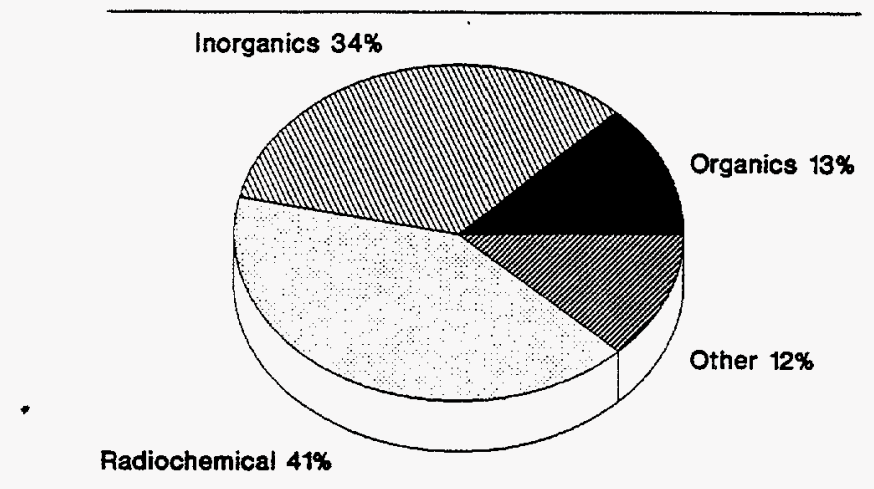

a) Organics

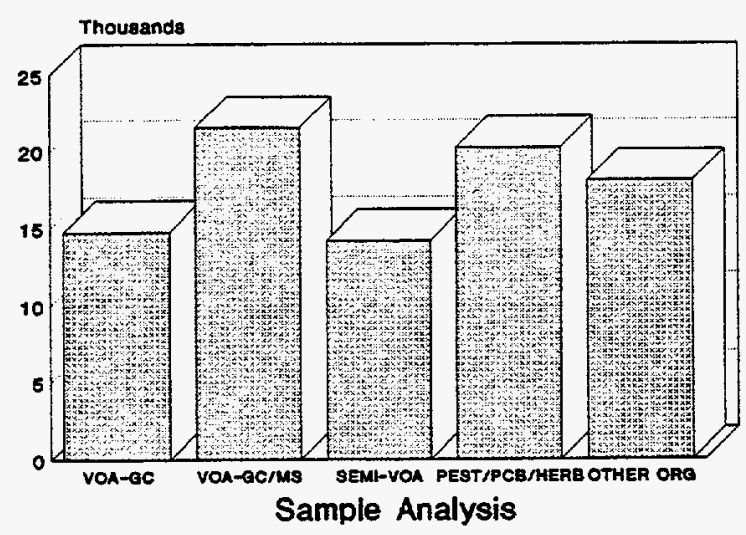

c) Radiochemical

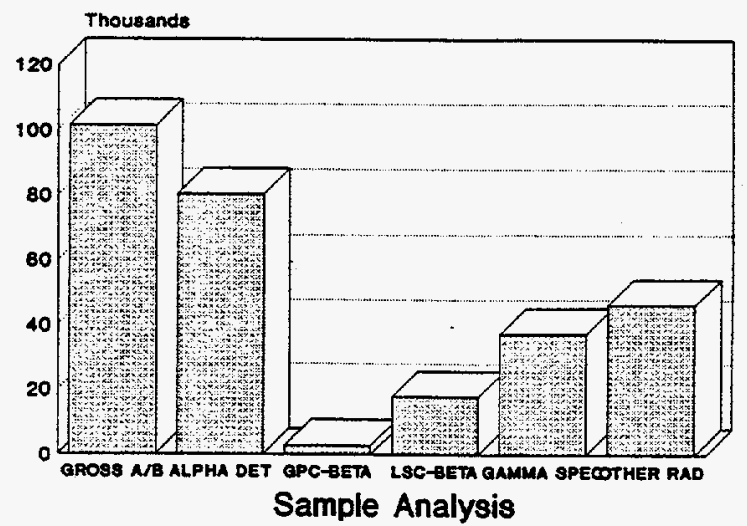

b) Inorganics

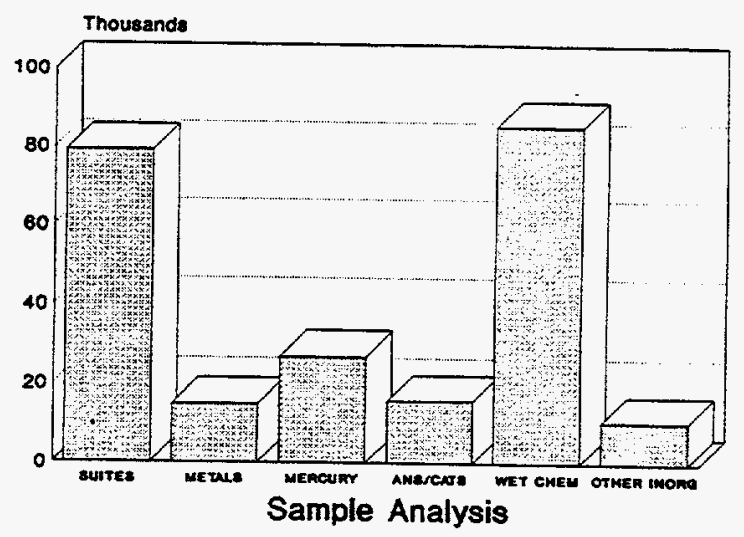

d) Other

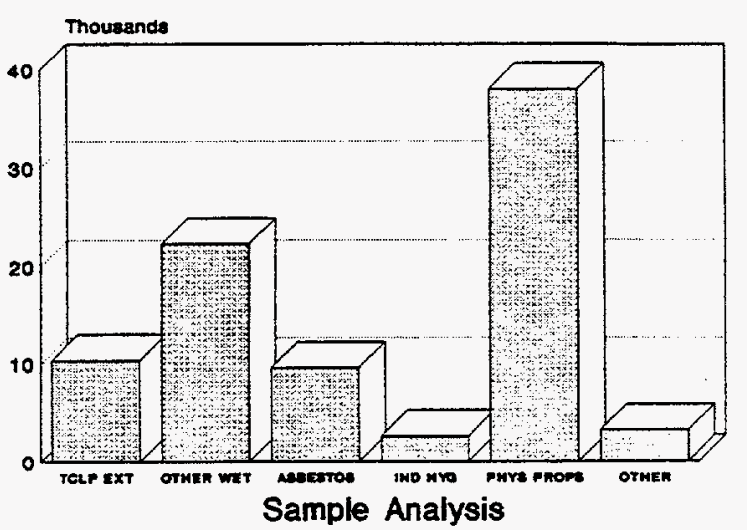

Figure 18: Distribution of FY 93 Needs by Sample Analysis Type 


\section{FY 94}

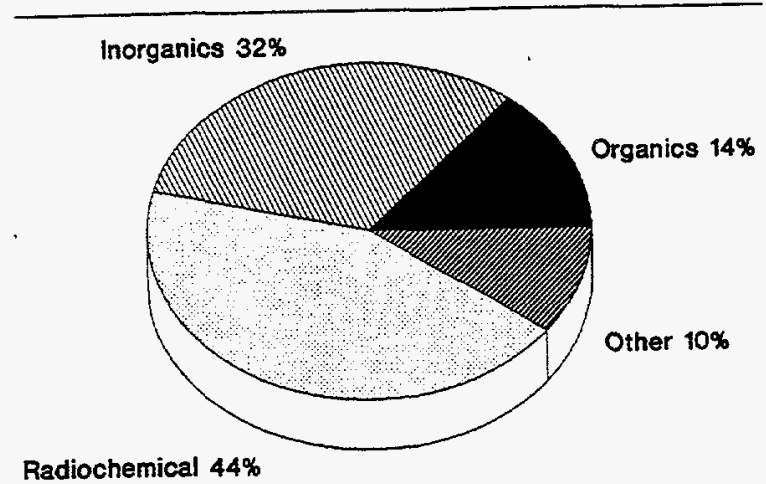

a) Organics

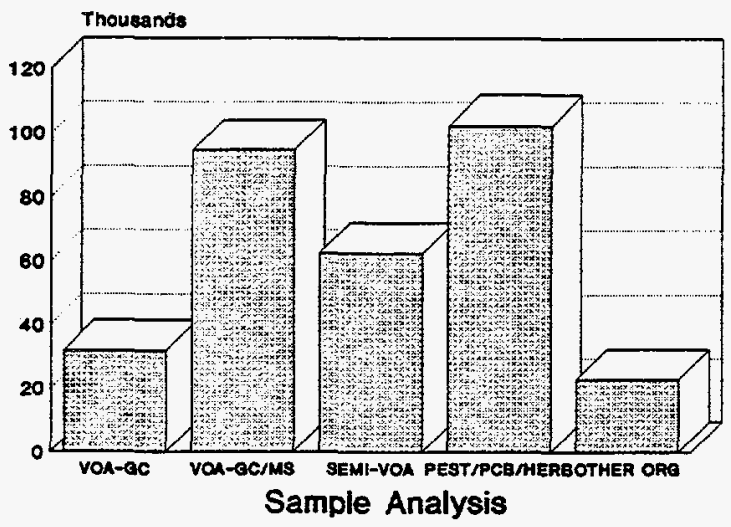

c) Radiochemical

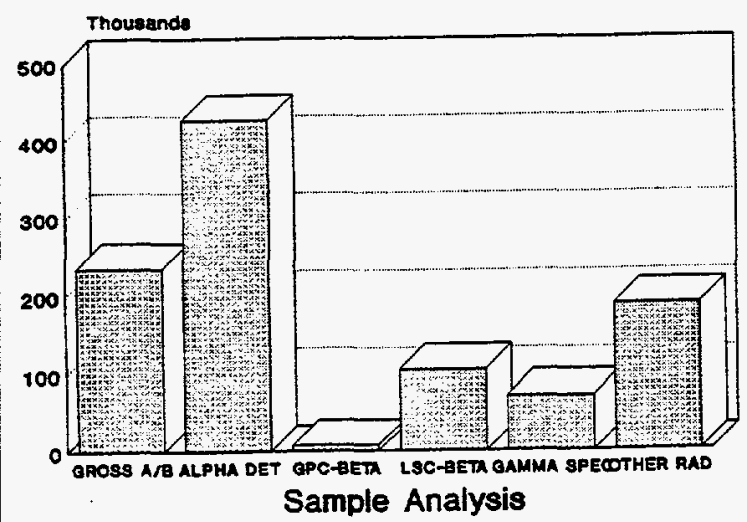

b) Inorganics

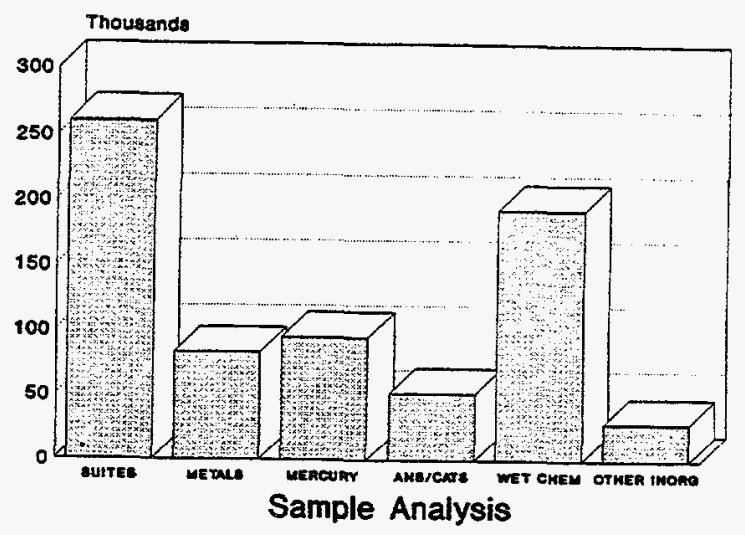

d) Other

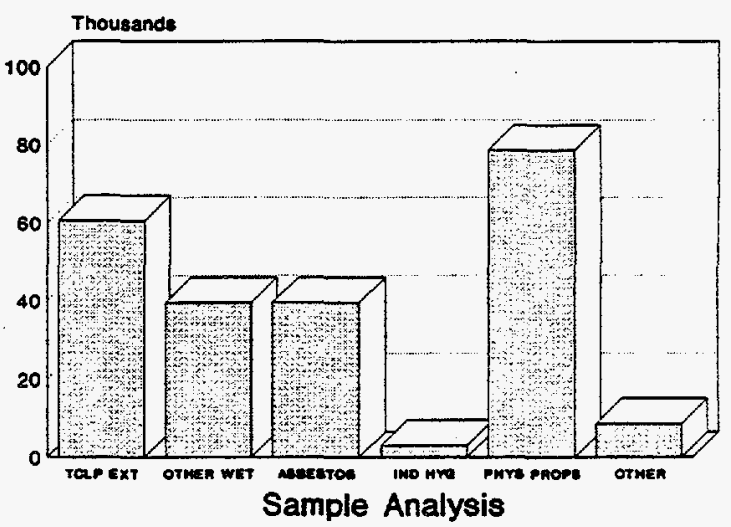

Figure 19: Distribution of FY 94 Needs by Sample Analysis Type 


\section{FY 95-99}

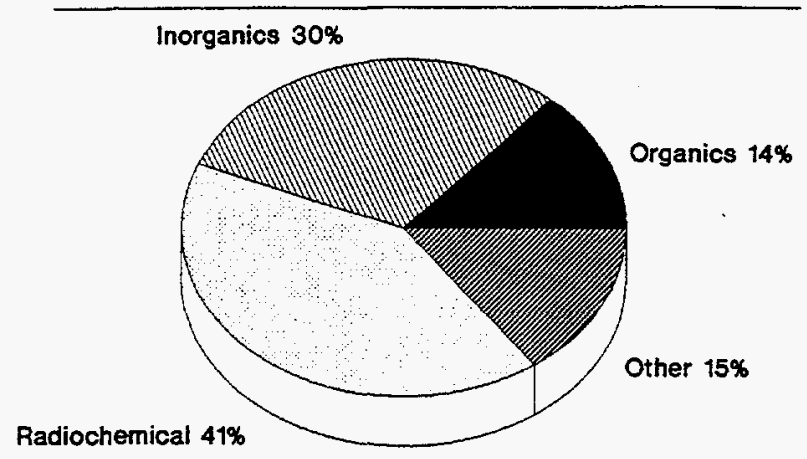

a) Organics

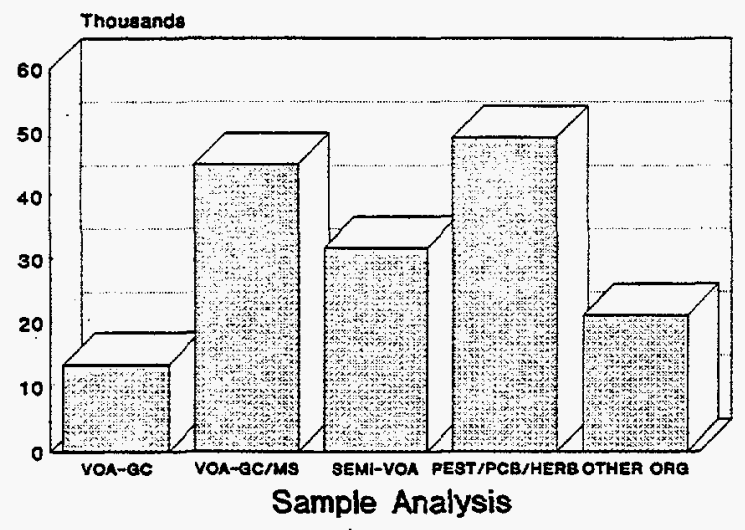

c) Radiochemical

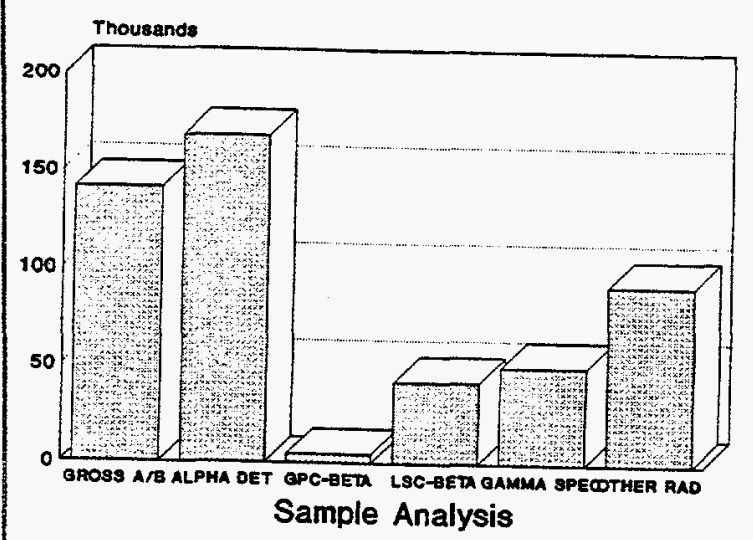

b) Inorganics

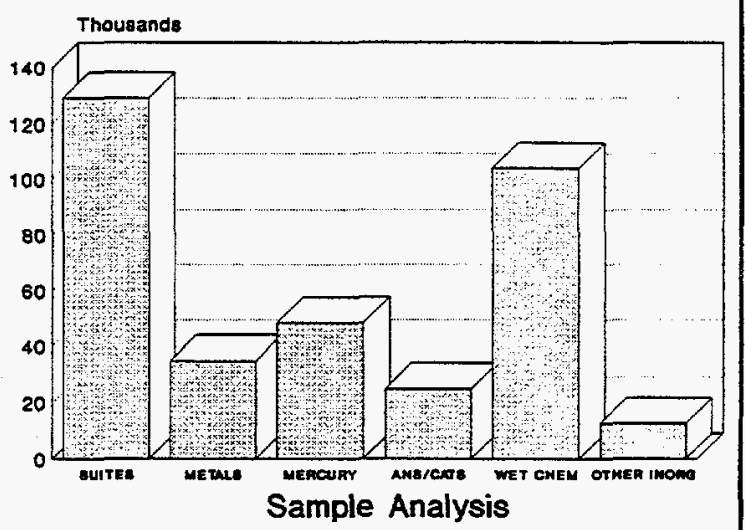

d) Other

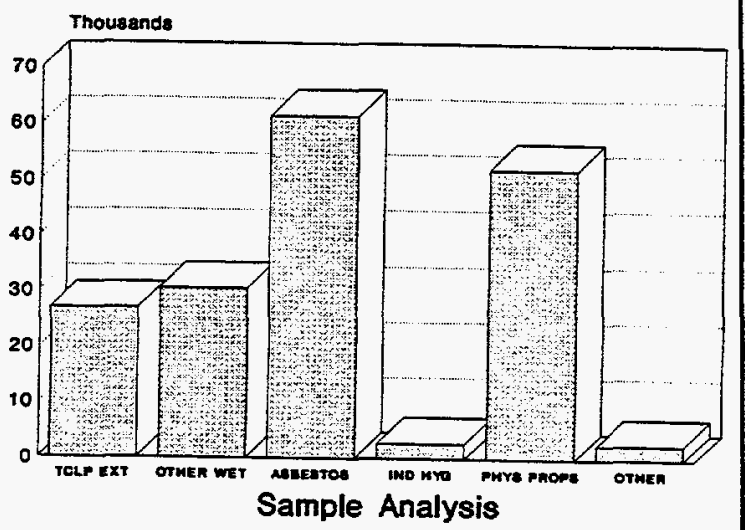

Figure 20: Distribution of FY 95-99 Needs by Sample Analysis Type 
projected distribution of sample analyses by type remains similar in the FY 93-99 time period. Most of the radiochemical sample analyses are gross alpha/beta and alpha spectroscopy measurements, and most of the inorganic sample analyses involve analyzing suites of metals and wet chemical measurements. Projections of organic sample analyses are distributed approximately evenly between all the sample analysis types.

\subsection{Conclusions}

An attempt was made in this study to project EM analytical needs for the period FY 94-99 based on data obtained from the field for FY 93 and from planned activities. The reliability of the results compiled from the data collected is as high as possible, given that field data were received from sites representing only about 40 percent of the DOE Complex, and verification of the data received on both FY 93 analytical demand and planned activities could not be performed. With these limitations, however, a scenario of EM analytical demand was made. The clear features of this scenario include a forecast of an increase in demand for FY 94 and FY 95, followed by a reduction and leveling off of demand in the out-years. This data was collected in order to compare with DOE analytical capacity, and this comparison is made in the next section of the report. 


\subsection{COMPARISON OF DOE LABORATORY CAPACITY TO EM ANALYTICAL NEEDS PROJECTIONS}

\subsection{Introduction}

One of the principal objectives of the studies described in this report is to compare DOE laboratory capacity to EM analytical needs, and to identify any shortfalls or surpluses in laboratory capacity. To achieve this objective, equivalent sets of sample analyses must be used in evaluating both the EM analytical needs and the DOE laboratory capacity. This is described in Section 4.2, and is followed by comparisons of these equivalent sample analyses in Section 4.3.

\subsection{Standardization of Sample Analyses}

A standard set of sample analysis types was developed for making comparisons between DOE laboratory capacity and EM analytical demand. Such a common set could not be used in the original data calls, since laboratory capacity is determined in terms of instrument-based sample analyses, while analytical demand is evaluated in terms of analyte-based sample analyses. For many sample analyses these may be identical, but in some cases analysis of certain analytes may be performed by one of several instruments. Furthermore, a requestor of a sample analysis may neither know, nor even be concerned about the instrument used for analysis.

The common set of sample analyses was therefore developed based on the most probable instrumentation used for performing the most probable analytes. Table $\mathbf{X}$ presents the standard sample analyses equivalent to each sample analysis used in estimating DOE laboratory capacity, and Table XI presents the standard sample analyses equivalent to each sample analysis used in projecting EM analytical needs.

\subsection{Comparison of Standardized Sample Analyses}

Estimates of DOE analytical laboratory capacity and EM analytical demand were recalculated using the set of common sample analysis types. Comparison of the DOE laboratory capacity and EM analytical demand for each common sample analysis is shown in Table XII. Numbers for utilized, existing, and upgradable capacity are given together with projected numbers for the analytical demand in FY 93 through FY 99. Totals for all sample analysis types are given at the end of the table.

Converting the estimates of laboratory capacity and analytical demand to the common set of sample analyses is observed to increase the total numbers of sample analyses in both sets of estimates as compared to the numbers presented earlier in this report. This increase is much greater for the analytical demand numbers than for the laboratory capacity numbers. This is primarily because EM analytical demand for many inorganic sample analyses was reported previously in terms of suites of metals. Using the standardized set of sample analyses, each suite is reported as five sample analyses. 
Table X: Common Sample Analyses Equivalent to Sample Analyses Used in Estimating DOE Laboratory Capacity

\begin{tabular}{|c|c|}
\hline Capacity Term & Common Equivalent \\
\hline ORGANICS & ORGANICS \\
\hline $\mathrm{VOA}-\mathrm{GC}$ & VOA-GC \\
\hline VOA-GC/MS & VOA-GC/MS \\
\hline SEMI-VOA & SEMI-VOA \\
\hline PEST & PEST/HERB/PCB \\
\hline HERB & PEST/HERB/PCB \\
\hline PCB & PEST/HERB/PCB \\
\hline PCB/PEST & PEST/HERB/PCB \\
\hline OTHER-ORG & OTHER-ORG \\
\hline INORGANICS & INORGANICS \\
\hline ICP-AES METALS & ICP-AES METALS \\
\hline ICP-MS METALS & ICP-MS METALS \\
\hline GF-AA METALS & GF-AA METALS \\
\hline MERCURY & MERCURY \\
\hline CYANIDE & ANIONS/CATIONS \\
\hline ANIONS/CATIONS & ANIONS/CATIONS \\
\hline WET CHEM & WET CHEM \\
\hline OTHER-INORG & OTHER-INORG \\
\hline RADIOACTIVES & RADIOACTIVES \\
\hline GROSS ALPHAVBETA & GROSS ALPHA/BETA \\
\hline ALPHA DET-Np237 & ALPHA DET - Np \\
\hline ALPHA DET-Pu,Am & ALPHA DET-Pu + ALPHA DET-Am \\
\hline ALPHA DET-PU & ALPHA DET $-\mathrm{Pu}$ \\
\hline ALPHA DET -U & ALPHA DET -U \\
\hline ALPHA DET-Am & ALPHA DET-Am \\
\hline ALPHA DET-Np & ALPHA DET -NP \\
\hline ALPHA DET - Th & ALPHA DET -Th \\
\hline ALPHA DET-U,Th, Pu & ALPHA DET-U + ALPHA DET-Th + ALPHA DET-PU \\
\hline ALPHA DET - U235 & ALPHA DET $-U$ \\
\hline ALPHA DET-PU, U, Am & ALPHA DET - PU + ALPHA DET -U + ALPHA DET - Am \\
\hline ALPHA DET - U, Np, Pu,Am & ALPHA DET-U + ALPHA DET - Np + ALPHA DET - Pu + ALPHA DET-Am \\
\hline ALPHA DET-Ra226 & ALPHA DET-Ra \\
\hline ALPHA DET-Ra228 & ALPHA DET-Ra \\
\hline ALPHA SPEC & ALPHA SPEC \\
\hline GPC-BETA-Pb210 & GPC-BETA-Pb210 \\
\hline GPC-BETA-Sr90 & GPC-BETA-Sr90 \\
\hline GPC-BETA-Pm147 & GPC-BETA-Pm147 \\
\hline GPC-BETA-Se79 & GPC-BETA-Se79 \\
\hline LSC-BETA-H3 & LSC-BETA-H3 \\
\hline LSC-BETA-Rn222 & LSC-BETA-Rn222 \\
\hline LSC-BETA- 1129 & LSC-BETA-I129 \\
\hline LSC-BETA-C14 & LSC-BETA-C14 \\
\hline LSC-BETA-TC99 & LSC-BETA-TC99 \\
\hline LSC-BETA & LSC-BETA \\
\hline GAMMA SPEC & GAMMA SPEC \\
\hline OTHER-RAD & OTHER-RAD \\
\hline OTHEA & OTHER \\
\hline TCLPEXTRACTION & TCLP EXTRACTION \\
\hline OTHER-PREP & OTHEA \\
\hline OTHER-EXPLO & OTHER \\
\hline OTHEF-BIO & OTHER \\
\hline OTHER-PHYS & PHYS PROPS \\
\hline OTHER & OTHER \\
\hline
\end{tabular}



Table XI: Common Sample Analyses Equivalent to Sample Analyses Used in
Projecting EM Analytical Needs

\begin{tabular}{|c|c|}
\hline Needs Term & Common Equivalent \\
\hline ORGANICS & ORGANICS \\
\hline Volatiles by GC/MS & VOA-GC/MS \\
\hline Volatiles by GC & NOA-GC \\
\hline Semi-Volatiles & SEMI-VOA \\
\hline Herbicides/Pesticides/PCBs & PEST/HERB/PCB \\
\hline Other Organics & OTHER-ORG \\
\hline INORGANICS & INORGANICS \\
\hline RCRA Appendix IX Metals & ICP-AES METALS + 3XGF-AA METALS + MERCURY \\
\hline CAM (California) Metals (RCRA) & ICP-AES METALS + 3XGF-AA METALS + MERCURY \\
\hline RCRA TCLP Metals and EPTOX Metals & ICP-AES METALS + 3XGF-AA METALS + MERCUAY \\
\hline EPA CLP Metals (CERCLA) & JCP-AES METALS + 3XGF-AA METALS + MERCURY \\
\hline NPDES Metals (Clean Water Act) & ICP-AES METALS + 3xGF-AA METALS + MERCURY \\
\hline Drinking Water Metals (SDWA) & ICP-AES METALS + 3XGF-AA METALS + MERCURY \\
\hline Metals by Other Regulatory Suite & ICP-AES METALS + 3XGF-AA METALS + MERCURY \\
\hline Metals by Suite, Other & ICP-AES METALS + 3XGF-AA METALS + MERCURY \\
\hline Metals by Metal Except Mercury & GF-AA METALS \\
\hline Mercury & MERCURY \\
\hline Anions/Cations-IC & ANIONS/CATIONS \\
\hline Inorganics Wet Chemistry & WET CHEM \\
\hline Other Inorganics & OTHER-INORG \\
\hline RADIOACTIVES & TRADIOACTIVES \\
\hline Gross Alpha/Beta & GROSS ALPHA/BETA \\
\hline Gamma Scan & GAMMA SPEC \\
\hline Transuranic & 2XALPHA SPEC \\
\hline Plutonium lsotopes & ALPHA DET-PU \\
\hline Uranium Isotopes & ALPHA DET $-U$ \\
\hline Curium Isotopes & ALPHA DET-Cm \\
\hline Thorium isotopes & ALPHA DET-Th \\
\hline Radium Isotopes & ALPHA DET-Ra \\
\hline Americium-241 & ALPHA DET-Am \\
\hline Neptunium - 237 & ALPHA DET - Np \\
\hline Polonium -210 & ALPHA DET-PO \\
\hline Tritium & LSC-BETA-H3 \\
\hline Radon-222 & LSC-BETA-Rn222 \\
\hline Lead-210 & GPC-BETA-Pb210 \\
\hline Carbon-14 & LSC-BETA-C14 \\
\hline Nickel-63 & $\angle S C-B E T A-N i 63$ \\
\hline Selenium-79 & GPC-BETA-Se79 \\
\hline Technetium-99 & LSC-BETA-TC99 \\
\hline Strontium Isotopes & GPC-BETA-Sr89+GPC-BETA-Sr90 \\
\hline Strontium -89 & GPC-BETA-Sr89 \\
\hline Strontium-90 & GPC-BETA-Sr90 \\
\hline lodine -129 & LSC-BETA- 1129 \\
\hline Nickel-59 & LSC-BETA-Ni59 \\
\hline Total Uranium & ICP-AES METALS \\
\hline Total Thorium & ICP-AES METALS \\
\hline Total Strontium & ICP-AES METALS \\
\hline Other RAD & OTHER-RAD \\
\hline OTHER & OTHER \\
\hline Other Wet Chemistry & OTHER \\
\hline TCLP Extraction & TCLP EXTRACTION \\
\hline Asbestos & PHYS PROPS \\
\hline Industrial Hygiene, NOS & OTHER \\
\hline Physical Properties & PHYS PROPS \\
\hline Other, Not Otherwise Specified (NOS) & OTHER \\
\hline
\end{tabular}


Table XII: Comparison of DOE Laboratory Capacity and EM Analytical Demand (in Thousands of Sample Analyses) by Sample Analysis Type

\begin{tabular}{|c|c|c|c|c|c|c|c|c|c|c|}
\hline \multirow{2}{*}{$\begin{array}{l}\text { Cap Type-Needs } \overline{F Y} \\
\text { Samp Anal } \\
\end{array}$} & \multicolumn{3}{|c|}{ CAPACITY } & \multicolumn{7}{|c|}{ NEEDS } \\
\hline & UC & $E C$ & MC & 93 & 94 & 95 & 96 & 97 & 98 & 99 \\
\hline & & & & & & & & & & \\
\hline VOA-GC & 3 & 3 & 4 & 15 & 32 & 31 & 9 & 9 & 10 & 9 \\
\hline VOA-GC/MS & 24 & 34 & 110 & 22 & 95 & 89 & 35 & 34 & 35 & 33 \\
\hline SEMI-VOA & 7 & 9 & 41 & 14 & 62 & 59 & 26 & 25 & 25 & 24 \\
\hline PEST/HERB/PCB & 23 & 32 & 82 & 20 & 102 & 103 & 36 & 35 & 37 & 36 \\
\hline OTHER-ORG & 2 & 63 & 163 & 18 & 23 & 24 & 22 & 20 & 20 & 20 \\
\hline TOTAL ORGANICS & 59 & 140 & 400 & 89 & 314 & 306 & 128 & 122 & 127 & 122 \\
\hline & & & & & & & & & & \\
\hline ICP-AES METALS & 50 & 136 & 407 & 95 & 342 & 323 & 121 & 118 & 120 & 115 \\
\hline ICP-MS METALS & 15 & 44 & 114 & 0 & 0 & 0 & 0 & 0 & 0 & 0 \\
\hline GF-AA METALS & 40 & 93 & 366 & 253 & 863 & 814 & 332 & 322 & 328 & 316 \\
\hline MERCURY & 26 & 77 & 423 & 106 & 355 & 340 & 140 & 136 & 138 & 134 \\
\hline ANIONS/CATIONS & 27 & 187 & 424 & 16 & 52 & 49 & 20 & 19 & 19 & 19 \\
\hline WET CHEM & 1 & 9 & 17 & 86 & 193 & 188 & 84 & 81 & 86 & 81 \\
\hline OTHER-INORG & 67 & 223 & 649 & 11 & 29 & 26 & 9 & 9 & 9 & 9 \\
\hline TOTAL INORGANICS & 227 & 768: & 2400 & 566 & 1834 & 1741 & 706 & 685 & 701 & 673 \\
\hline & & & & & & & & & & \\
\hline GROSS ALPHA/BETA & 247 & 456 & 1688 & 101 & 236 & 220 & 124 & 121 & 122 & 120 \\
\hline ALPHADET-U & 22 & 33 & 75 & 25 & 123 & 117 & 37 & 36 & 36 & 35 \\
\hline ALPHADET-Np & 2 & 7 & 14 & 4 & 46 & 47 & 3 & 3 & 3 & 2 \\
\hline ALPHADET-PU & 22 & 41 & 161 & 20 & 48 & 41 & 26 & 26 & 26 & 25 \\
\hline ALPHA DET-Am & 10 & 25 & 132 & 17 & 38 & 32 & 23 & 23 & 23 & 22 \\
\hline ALPHADET-Cm & 0 & 0 & 0 & 1 & 2 & 2 & 0 & 0 & 0 & 0 \\
\hline ALPHADET - Th & 6 & 7 & 13 & 4 & 43 & 45 & 7 & 7 & 8 & 7 \\
\hline ALPHADET-Ra & 1 & 1 & 2 & 5 & 10 & 8 & 7 & 7 & 7 & 7 \\
\hline ALPHA DET-PO & 0 & 0 & $\overline{0}$ & 1 & 2 & 2 & 0 & 0 & 0 & 0 \\
\hline ALPHA SPEC & 14 & 26 & 181 & 4 & 115 & 119 & 5 & 5 & 4 & 4 \\
\hline GPC-BETA-Pb210 & 0 & 1 & 2 & 0 & 0 & 0 & 0 & 0 & 0 & 0 \\
\hline GPC-BETA-Sr89 & 0 & 0 & 0 & 요 & 요 & 0 & 0 & 0 & 0 & 0 \\
\hline GPC-BETA-Sr90 & 3 & 12 & 27 & 2 & $\overline{6}$ & 5 & 3 & 3 & 3 & 3 \\
\hline GPC-BETA-Pm 147 & 0 & 5 & 5 & 0 & 0 & 0 & $\overline{0}$ & 0 & 0 & 0 \\
\hline GPC-BETA-Se79 & 0 & 1 & 2 & 0 & 1 & 7 & 1 & 1 & 1 & 1 \\
\hline LSC-BETA-H3 & 22 & 28 & 77 & 13 & 31 & 24 & 19 & 18 & 18 & 18 \\
\hline LSC-BETA-Rn222 & 0 & 0 & 1 & 0 & 0 & 0 & 0 & 0 & 0 & 0 \\
\hline LSC-BETA-1129 & 0 & 1 & 8 & 1 & 2 & 2 & 1 & 1 & 1 & 1 \\
\hline LSC-BETA-C14 & 1 & 2 & 3 & 0 & 0 & 0 & 0 & 0 & $\overline{0}$ & 0 \\
\hline LSC-BETA-TC99 & 14 & 19 & 60 & 3 & 71 & 75 & 7 & 7 & 7 & 7 \\
\hline LSC-BETA-Ni59 & 0 & 0 & 0 & 0 & 0 & 0 & 0 & 0 & 0 & 0 \\
\hline LSC-BETA-Ni63 & 0 & 0 & 0 & 0 & 0 & 0 & 0 & 0 & 0 & 0 \\
\hline LSC-BETA & 1 & 3 & 3 & 0 & 0 & 0 & 0 & 0 & 0 & 0 \\
\hline GAMMA SPEC & 36 & 104 & 403 & 37 & 71 & 66 & 46 & 45 & 46 & 45 \\
\hline OTHER-RAD & 9 & 14 & 40 & 30 & 106 & 103 & 52 & 51 & 53 & 49 \\
\hline TOTAL RAD & 408 & 786 & 2897 & 270 & 952 & 910 & 364 & 355 & 358 & 349 \\
\hline & & & & & & & & & & \\
\hline TCLP EXTRACTION & 4 & 13 & 34 & 10 & 61 & 66 & 18 & 17 & 18 & 16 \\
\hline PHYS PROPS & 14 & 95 & 202 & 48 & 118 & 143 & 104 & 104 & 109 & 107 \\
\hline OTHER & 16 & 107 & 194 & 28 & 51 & 44 & 35 & 34 & 34 & 33 \\
\hline TOTAL OTHER & $\overline{34}$ & 215 & 431 & 86 & 230 & 253 & 157 & 155 & 161 & 157 \\
\hline & & & & & & & & & & \\
\hline TOTAL SAMP ANALS & 728 & 1909 & 6127 & 1010 & 3330 & 3211 & 1355 & 1317 & 1348 & 1301 \\
\hline
\end{tabular}

Total utilized DOE laboratory capacity is estimated to be about 730,000 standard sample analyses compared to an EM analytical demand of around one million sample analyses for FY 93. This demand is projected to increase to over three million sample analyses in FY 94 and FY 95 before decreasing to around 1.3 million sample analyses 
per year in the subsequent years. Total existing DOE laboratory capacity is estimated to be about 1.9 million sample analyses, and this may be upgraded to a capacity of over six million sample analyses. It is re-emphasized that the uncertainty in the numbers provided here may be very large, especially for those given for EM analytical demand, and all conclusions drawn from these numbers should be considered very tentative.

It should be noted that EM analytical demand is currently being met also by laboratories in the commercial sector, for which a capacity has not been estimated, It should be further noted that DOE analytical laboratories provide analytical services not only to EM, but also to other DOE customers. It is therefore clear that while the utilized capacity of DOE laboratories is insufficient to meet the projected analytical demand of EM, the laboratories have an existing capacity that can meet the demand, except for FY 94 and FY 95, when a surge in analytical demand is projected. Upgrades to the DOE laboratories would be needed if these laboratories are to be the sole source of analytical services for EM in this time period.

On observing these numbers more closely, comparisons may be made for each standard sample analysis type. It may be seen that, in general, for organic, inorganic, and other sample analyses, $U C$ is about half of, and $E C$ is about equal to the average EM analytical needs over the FY 93 through FY 99 period. Of course, in the two peak years of EM analytical demand, these capacities represent a considerably smaller fraction of the analytical demand. For all radiochemical sample analyses, UC appears to be about sufficient to meet the average EM analytical demand. However, this total number is dominated by gross alpha/beta sample analyses, which represent over half of the total DOE radiochemical sample analysis capacity, and for which DOE laboratory capacity is sufficient to meet EM demand for every FY modelled. The high capacity for gross alpha/beta sample analyses is needed for screening all sample suspected of having radioactive contamination, especially those shipped to the commercial sector. The remaining radiochemical sample analyses show a similar pattern to all the other sample analyses on comparing the DOE laboratory capacity to EM analytical demand.

Comparison of the DOE laboratory capacity to EM analytical demand according to the radiation levels and matrix types of the samples that are analyzed is shown in Table XIII. For the RO and R1 categories, there is not enough utilized DOE laboratory capacity to meet EM demand, and even at the EC level there is barely enough capacity to meet demand in the period FY 96 through FY 99, when EM analytical demand is expected to flatten out. Sample analyses performed on these type of samples, however, are those most easily carried out by laboratories in the commercial sector. For the higher radiation level samples in the R2 through R4 categories, UC is currently not able to meet the anticipated demand. EC can, however, meet this demand even in the years when demand is expected to surge. The higher radiation level samples are currently being analyzed at the DOE M\&O laboratories, and the laboratories claim that they are easily able to gear up to their EC levels for such samples. 
Table XIII: Comparison of DOE Laboratory Capacity and EM Analytical Demand (in Thousands of Sample Analyses) by Sample Radiation Level and Matrix Type

\begin{tabular}{|c|c|c|c|c|c|c|c|c|c|c|}
\hline \multirow{2}{*}{$\begin{array}{l}\text { Cap Type-Needs FY } \\
\text { Rad-Matrix }\end{array}$} & \multicolumn{3}{|c|}{ CAPACITY } & \multicolumn{7}{|c|}{ NEEDS } \\
\hline & UC & EC & MC & 93 & 94 & 95 & 96 & 97 & 98 & 99 \\
\hline & & & & & & & & & & \\
\hline RO & 213 & 502 & 1551 & 643 & 1241 & 1065 & 757 & 736 & 750 & 725 \\
\hline Ro/R1 & 238 & 586 & 1530 & & & & & & & \\
\hline R1 & 255 & 357 & 1661 & 411 & 1783 & 1831 & 633 & 614 & 631 & 618 \\
\hline$\overline{\mathrm{R} 2}$ & 15 & 431 & 1208 & 26 & 363 & 368 & 24 & 24 & 23 & 22 \\
\hline$\overline{R 3}$ & 6 & 23 & 102 & 4 & 14 & 20 & 20 & 23 & 23 & 15 \\
\hline R4 & 1 & 10 & 76 & 4 & 5 & 5 & 5 & 5 & 5 & 5 \\
\hline & & & & & & & & & & \\
\hline M1 & 389 & 1117 & 3637 & 466 & 1311 & 1237 & 593 & 578 & 575 & 568 \\
\hline $\mathrm{M} 2$ & 276 & 458 & 1514 & 268 & 934 & 837 & 530 & 508 & 525 & 512 \\
\hline M3 & 62 & 333 & 976 & 198 & 1009 & 1058 & 148 & 147 & 164 & 136 \\
\hline
\end{tabular}

The distribution of DOE laboratory capacity and EM analytical demand between the three matrix types of samples are similar for most of the capacity types and needs fiscal years. In both cases, the capacity and needs are greater for sample analyses performed on $\mathrm{M} 1$ and $\mathrm{M} 2$ samples than for those performed on M3 samples. There is, however, a relatively high fraction of M3 samples that require analysis during the two years FY 94 and FY 95, compared to the other years. Since M3 samples are the most difficult to analyze, this will only exacerbate any shortfall in laboratory capacity during these years.

Comparisons were also made between the regulatory protocols used for performing the sample analyses making up the DOE laboratory capacity and the regulatory drivers of the EM analytical needs. These comparisons are shown in Table XIV. While the DOE laboratory capacity is about the same order of magnitude for SW-846 sample analyses as the EM demand for RCRA sample analyses, and the same is true for Clean Water Act (CWA) sample analyses, demand far outstrips the capacity for CERCLAdriven sample analyses performed by CLP protocols. This is probably because such sample analyses are predominantly performed by laboratories in the commercial sector, and it appears that this strategy will be necessary also in future years.

As noted in Section 2.0, just over half the DOE laboratory capacity is currently being utilized for EM sample analyses, and this data is presented again in Table XIV for the standardized sample analyses. On making any comparisons between DOE laboratory capacity and EM analytical needs, it should always be remembered that EM is not necessarily the sole client for the DOE laboratories. However, if laboratories are able to work closer to their existing capacities, the EM fraction of their workload will increase substantially.

A final comparison of DOE laboratory capacity to EM analytical demand is made in Table $\mathbf{X V}$, in which the sample analysis numbers are presented according to laboratories and sites. For those sites that reported to the call for data for the EM analytical needs assessment, the needs are generally somewhat greater than the local DOE Management and Operations (M\&O) laboratory capacity with some notable 
Table XIV: Comparison of DOE Laboratory Capacity and EM Analytical Demand (in Thousands of Sample Analyses) by Regulatory Driver

\begin{tabular}{|c|c|c|c|c|c|c|c|c|c|c|c|}
\hline \multirow{2}{*}{$\begin{array}{l}\text { Cap Typel } \\
\text { Reg Protocol-Dist'n }\end{array}$} & \multicolumn{3}{|c|}{ CAPACTTY } & \multirow{2}{*}{$\begin{array}{l}\text { Meeds FY/ } \\
\text { Regulatory Driver }\end{array}$} & \multicolumn{7}{|c|}{ NEEDS } \\
\hline & $\mathbf{U C}$ & $\mathrm{EC}$ & MC & & 93 & 94 & 95 & 96 & 97 & 98 & 99 \\
\hline & & & & & & & & & & & \\
\hline SW846 & 168 & 580 & 1663 & RCRA & 243 & 1616 & 1716 & 389 & 389 & 404 & 401 \\
\hline CLP & 6 & 20 & 76 & CERCLA & 141 & 250 & 231 & 170 & 143 & 168 & 136 \\
\hline CWA & 83 & 142 & 437 & CWA & 57 & 71 & 74 & 73 & 73 & 73 & 72 \\
\hline NIOSH & 36 & 40 & 105 & NPDES & 2 & 2 & 2 & 2 & 2 & 2 & 2 \\
\hline Other Reg & 312 & 569 & 1727 & NEPA & 1 & 7 & 7 & 7 & 7 & 7 & 7 \\
\hline Waste Treatment & 10 & 27 & 240 & CAA & 12 & 13 & 14 & 13 & 13 & 13 & 13 \\
\hline Prod'n Support & 37 & 358 & 1299 & TSCA & 36 & 111 & 115 & 115 & 114 & 113 & 113 \\
\hline Other Non-Reg & 77 & 175 & 580 & STATE & 138 & 148 & 153 & 152 & 151 & 151 & 151 \\
\hline & & & & OTHER & 380 & 1111 & 899 & 431 & 423 & 413 & 404 \\
\hline$E M$ & 391 & 1542 & 4357 & & & & & & & & \\
\hline Non-EM & 338 & 367 & 1770 & & & & & & & & \\
\hline
\end{tabular}

exceptions. For example, the needs of the Oak Ridge sites appear to be less than the capacity of their laboratories, except for the Oak Ridge K-25 site in FY 94 and FY 95 , when demand is projected to grow more than tenfold. While the Oak Ridge Y-12 laboratory has the largest reported UC in the DOE Complex, the EM analytical demand from the Oak Ridge Y-12 site alone is projected to be about two orders of magnitude smaller. In the case of Fernald, while EM demand is currently much greater than the capacity at that site, this demand is projected to decrease significantly to levels below even the currently utilized capacity in FY 96 and beyond.

\subsection{Conclusions}

In this section an attempt was made to compare data collected for assessing EM's analytical needs with data collected on DOE laboratory capacity. While only general comparisons may be made, since the data is not considered to be highly reliable, some conclusions may be drawn. This effort has demonstrated that such comparisons may be made using a sample analysis as the common unit, and these comparisons provide detailed information on when, where, and for which type of samples and sample analyses there may be surpluses or shortfalls in DOE laboratory capacity necessary to meet EM's analytical needs. It is evident from this initial set of studies that DOE capacity alone is unable to meet all EM's analytical needs unless there is major upgrading and more effective use of the existing capacity. 
Table XV: Comparison of DOE Laboratory Capacity and EM Analytical Demand (in Thousands of Sample Analyses) by Laboratory and Site

\begin{tabular}{|c|c|c|c|c|c|c|c|c|c|c|c|}
\hline \multirow{2}{*}{$\begin{array}{l}\text { Cap Typel } \\
\text { Laboratory }\end{array}$} & \multicolumn{3}{|c|}{ CAPACITY } & \multirow{2}{*}{$\begin{array}{l}\text { Needs FYI } \\
\text { Site }\end{array}$} & \multicolumn{7}{|c|}{ NEEDS } \\
\hline & UC & $\mathbf{E C}$ & $\mathrm{MC}$ & & 93 & 94 & 95 & 96 & 97 & 98 & 99 \\
\hline & & & & & & & & & & & \\
\hline Grand Junction & 10 & 54 & 109 & & & & & & & & \\
\hline Los Alamos Nat' | Lab & 37 & 34 & 277 & & & & & & & & \\
\hline Argonne East & 5 & 13 & 70 & & & & & & & & \\
\hline Argonne West & 2 & 0 & 0 & & & & & & & & \\
\hline Brookhaven Nat'/ Lab & 12 & 0 & 0 & & & & & & & & \\
\hline Fernald & 39 & 85 & 254 & Fernald & 105 & 165 & 163 & 16 & 15 & 24 & 14 \\
\hline INEL - ACU & 0 & 1 & 5 & & & & & & & & \\
\hline INEL - B\&W & 1 & 10 & 0 & & & & & & & & \\
\hline INEL - WINCO & 0 & 25 & 58 & & & & & & & & \\
\hline Total INEL & 2 & 36 & 63 & INEL & 49 & 52 & 58 & 53 & 48 & 54 & 54 \\
\hline West Valley & 4 & 4 & 4 & & & & & & & & \\
\hline Nevada Test Site & 29 & 39 & 71 & Nevada Test Site & 2 & 49 & 70 & 70 & 70 & 71 & 70 \\
\hline Oak Ridge - K25 & 41 & 80 & 325 & Oak Ridge - K25 & 25 & 517 & 517 & 46 & 45 & 41 & 42 \\
\hline Oak Ridge Nat'l Lab & 30 & 37 & 59 & Oak Ridge Nat'l Lab & 21 & 34 & 29 & 22 & 21 & 20 & 20 \\
\hline Oak Ridge $-Y_{12}$ & 221 & 312 & 1031 & Oak Ridge - Y12 & 7 & 7 & 6 & 7 & 6 & 6 & 4 \\
\hline Total Oak Ridge Res'n & 292 & 429 & 1415 & Total Oak Fidge Res'n & 53 & 558 & 553 & 75 & 72 & 68 & 66 \\
\hline Paducah & 60 & 60 & 152 & & & & 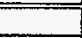 & & & & \\
\hline Portsmouth & 80 & 86 & 203 & & & & & & & & \\
\hline Hanford - WHC 222S & 38 & 644 & 1548 & & & & & & & & \\
\hline Hanford - PNL 325 & 8 & 63 & 273 & & & & & & & & \\
\hline Total Hanford & 46 & 707 & 1821 & & & & & & & & \\
\hline Rocky Flats $-123 \mathrm{Lab}$ & 47 & 246 & 630 & & & & & & & & \\
\hline Rocky Flats - GL881 & 15 & 16 & 87 & & & & & & & & \\
\hline Rocky Flats - RFPA La & 6 & 17 & 95 & & & & & & & & \\
\hline Total Rocky Flats & 68 & 279 & 813 & Rocky Flats & 87 & 277 & 175 & 162 & 159 & 159 & 156 \\
\hline WSAC-ALD-CLAB & 6 & 20 & 728 & & & & & & & & \\
\hline WSRC-ALD-320M & 0 & 1 & 0 & & & & & & & & \\
\hline WSRC-ALD Water & 6 & 9 & 23 & & & & & & & & \\
\hline WSRC-ALD-ETF & 1 & 0 & 0 & & & & & & & & \\
\hline WSRC-ALD-Tritium & 1 & 3 & 3 & & & & & & & & \\
\hline WSRC-ALD - ITP & 1 & 0 & 0 & & & & & & & & \\
\hline WSRC-SRTC-ADS & 23 & 41 & 112 & & & & & & & & \\
\hline WSRC-SRTC-DWPT & 5 & 8 & 8 & & & & & & & & \\
\hline Total Savannah River & 44 & 82 & 874 & Savannah River & 93 & 97 & 96 & 96 & 97 & 97 & 97 \\
\hline & & & & & & & & & & & \\
\hline & & & & $E M-30$ & 372 & 462 & 459 & 414 & 422 & 412 & 404 \\
\hline & & & & $E M-40$ & 413 & 2647 & 2473 & 687 & 642 & 682 & 646 \\
\hline & & & & $E M-60$ & 167 & 212 & 186 & 186 & 187 & 187 & 187 \\
\hline Complex-wide & 728 & 1909 & 6127 & Complex-wide . & 1010 & 3330 & 3211 & 1355 & 1317 & 1348 & 1301 \\
\hline
\end{tabular}




\subsection{OVERALL CONCLUSIONS}

This report describes two studies that were performed by EM-263 to estimate DOE analytical laboratory capacity and to project EM analytical needs. It also includes the first attempt made to compare the data collected from the two studies in order to provide EM with an overall assessment of available capacity to meet its analytical needs. These efforts did suffer somewhat from incomplete reporting of data from the field, which led to final numbers with questionable reliability. However, the data collected provides. EM with a view of its general analytical needs and the DOE capacity available to meet them.

A clear conclusion from these studies is that the DOE analytical laboratories do not currently have all the capacity necessary to meet all of EM's analytical needs. It is evident that laboratories in the Commercial sector will have to continue to play a major role in meeting EM's analytical needs if no major upgrading of the DOE laboratory sector takes place. It is also clear, however, that effective sample management at both the field and the national level can allow the laboratories to make much more effective use of the capacity that is currently available. EM is making efforts in this direction through its development of a National Sample Management Program (NSMP) ${ }^{6}$ at EM headquarters, and Field Sample Management Programs (FSMPs) $^{7}$ at the Operations Offices.

Since such a concerted effort at estimating total EM analytical needs and DOE available laboratory capacity has not been attempted before, it could be expected that the data collected would not be of the highest quality or be the most complete. It is, however, encouraging to be able to obtain trends from the data and orders of magnitude for quantities that were hitherto unknown. There is, therefore, a need to continue these studies based on the experience gained so far using improved models and data collection techniques. 


\subsection{REFERENCES}

1. Preliminary Estimate of the Environmental Analytical Needs of the Department of Energy - prepared by the Future Analytical Support Team, December, 1991.

2. Report on Preliminary Estimate of EM Environmental Sample Analysis Needs, August 17, 1992.

3. EM-263 National Sample Tracking System, Version 1.0, October 15, 1993.

4. Quality Assurance Guidance for the Integrated Performance Evaluation Program in Support of EM Environmental Sampling and Analysis Activities, September 29, 1993.

5. Report on Hanford Laboratory Capacity Model, October, 1993.

6. Guidance for Establishment and Implementation of a National Sample Management Program in Support of EM Environmental Sampling and Analysis Activities, April 29, 1994.

7. Guidance for Establishment and Implementation of Field Sample Management Programs in Support of EM Environmental Sampling and Analysis Activities, April 29, 1994. 